PNNL-24275

U.S. DEPARTMENT OF

ENERGY

\title{
Supplemental Hazard Analysis and Risk Assessment - Hydrotreater
}

\author{
Peter P. Lowry \\ Katie A. Wagner
}

April 2015

Pacific Northwest

NATIONAL LABORATORY

Proudly Operated by Battelle Since 1965 


\title{
DISCLAIMER
}

This report was prepared as an account of work sponsored by an agency of the United States Government. Neither the United States Government nor any agency thereof, nor Battelle Memorial Institute, nor any of their employees, makes any warranty, express or implied, or assumes any legal liability or responsibility for the accuracy, completeness, or usefulness of any information, apparatus, product, or process disclosed, or represents that its use would not infringe privately owned rights. Reference herein to any specific commercial product, process, or service by trade name, trademark, manufacturer, or otherwise does not necessarily constitute or imply its endorsement, recommendation, or favoring by the United States Government or any agency thereof, or Battelle Memorial Institute. The views and opinions of authors expressed herein do not necessarily state or reflect those of the United States Government or any agency thereof.

\author{
PACIFIC NORTHWEST NATIONAL LABORATORY \\ operated by \\ BATTELLE \\ for the \\ UNITED STATES DEPARTMENT OF ENERGY \\ under Contract DE-AC05-76RL01830
}

Printed in the United States of America
Available to DOE and DOE contractors from the Office of Scientific and Technical Information,
P.O. Box 62, Oak Ridge, TN 37831-0062;
ph: (865) 576-8401
fax: $(865)$ 576-5728
email: reports@adonis.osti.gov

\begin{abstract}
Available to the public from the National Technical Information Service, U.S. Department of Commerce, 5285 Port Royal Rd., Springfield, VA 22161 ph: (800) 553-6847 fax: $(703) 605-6900$ email: orders@ntis.fedworld.gov online ordering: http://www.ntis.gov/ordering.htm
\end{abstract}

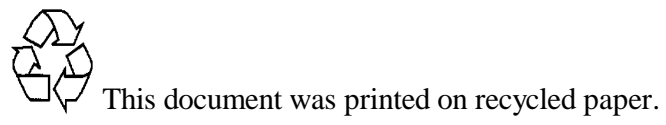


Prepared by:

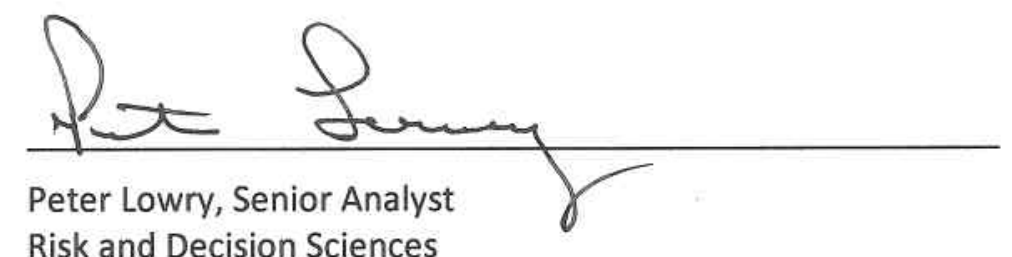

$\frac{4-27-15}{\text { Date }}$

Concurred by:

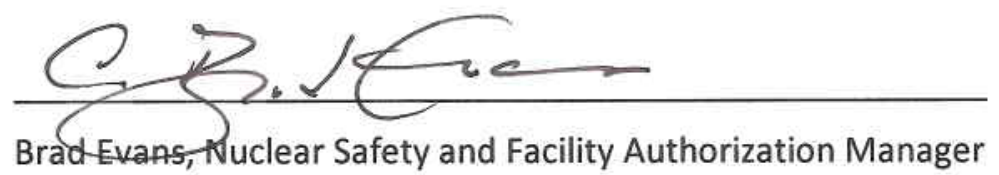

$4 / 27 / 15$

Date

Nuclear Operations Division

Ruck Otoh

$4 / 27 / 15$

Rick Orth, Technical Group Manager

Date

Chemical and Biological Process Development Group

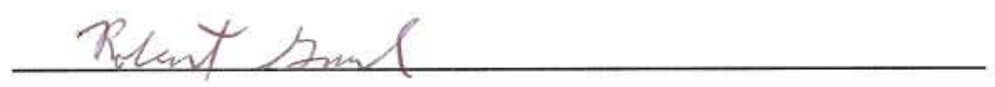

$4 / 27 / 15$

Robert Gruel, Capability Manager

Hydrotreater and Distillation Column Project

Date

Approved by:

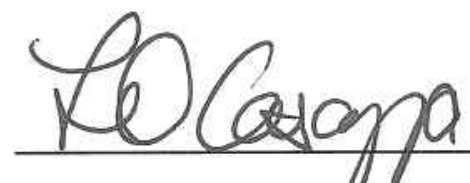

Larry Casazza, Chief Operating Officer

Energy and Environment Directorate

$4 / 27 / 15$

Date 
Supplemental Hazard Analysis and Risk Assessment - Hydrotreater

This page intentionally left blank 


\section{Executive Summary}

A supplemental hazard analysis was conducted and quantitative risk assessment performed in response to an independent review comment received by the Pacific Northwest National Laboratory (PNNL) from the U.S. Department of Energy Pacific Northwest Field Office (PNSO) against the Hydrotreater/Distillation Column Hazard Analysis Report issued in April 2013. The comment states:

“...based on our independent review and in light of our code responsibilities as the owner, we are directing Battelle to provide PNSO with the following items prior to start-up of the distillation column:

"3. Identification of prudent protective actions for the worker and the public (e.g., setback distances, shielding, procedures for minimizing time in the higher risk areas during operation, etc.) based on quantitative analysis and practical risk minimization approaches (for the distillation column).

Items 1 and 3 are also expected to be provided to PNSO for the hydrotreater unit prior to its operation..." (Emphasis added)

The supplemental analysis used the hazardous conditions documented by the previous April 2013 report as a basis. The conditions were screened and grouped for the purpose of identifying whether additional prudent, practical hazard controls could be identified, using a quantitative risk evaluation to assess the adequacy of the controls and establish a lower level of concern for the likelihood of potential serious accidents. Calculations were performed to support conclusions where necessary.

Additional controls were identified where the quantitative analysis demonstrated a need for additional preventive or mitigative measures. These include:

1. Modification of the safe operating procedure (SOP) to state that 1 ) any leak testing of the system (with nitrogen) will be conducted with the ventilation system operational and 2) the nitrogen system will be closed when not in use. This mitigates the presence of an oxygen deficient atmosphere during maintenance activities and non-operational timeframes. [Action Completed as part of Distillation Column Supplemental Hazard Analysis and Risk Assessment].

2. Modification of the SOP to require the operator to minimize the stay time within the enclosure during hydrotreater operations. This decreases the time an operator is subject to any incidents that might occur within the enclosure. [Action Completed as part of Distillation Column Supplemental Hazard Analysis and Risk Assessment].

3. Administrative controls to check relief header - Modify the SOP to require verification that the low pressure relief line is free of obstruction (e.g., mud dauber nest) (U-7 bounding event).

The following additional follow-on action items were identified during the analysis to verify assumptions or enhance worker safety:

1. During startup testing, ensure that valves fail in the appropriate direction, based on their credited function, on loss of air. 
2. Review areas of operator proximity to high pressure/high temperature system components to ensure adequate personal protective equipment (PPE) is provided against flashing steam release.

These additional hazard controls are prudent, practical measures to reduce risk to workers and the public. They will be implemented prior to operation of the hydrotreater unit. 


\section{Contents}

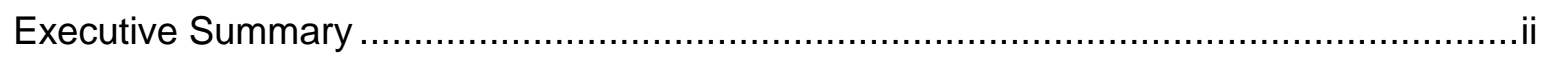

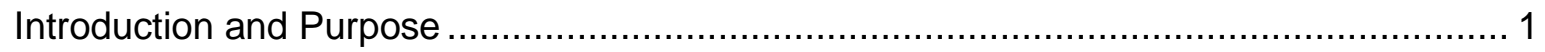

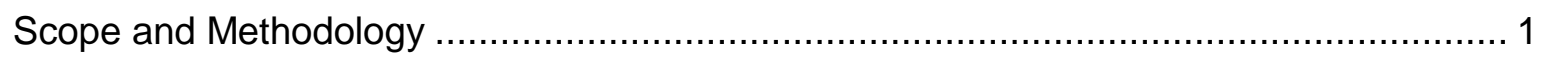

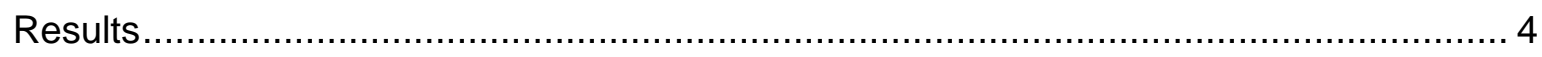

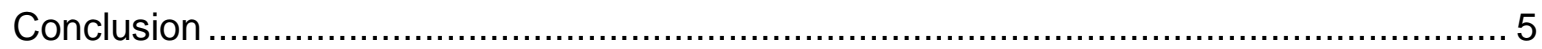

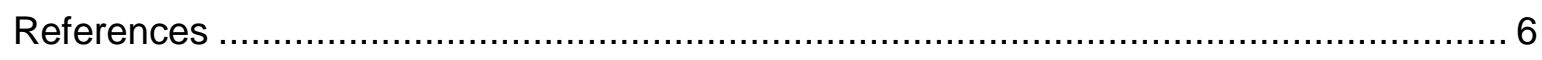

Table 1. Frequency Values and Data Sources .................................................. 8

Table 2. Supplemental Hazard Analysis and Risk Assessment Evaluation -

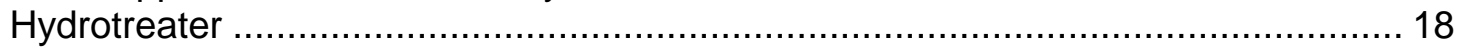

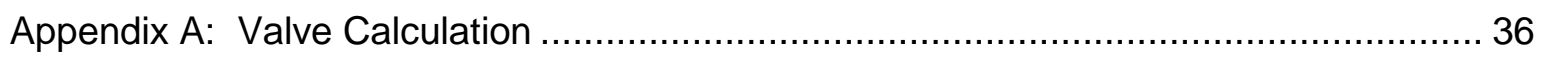

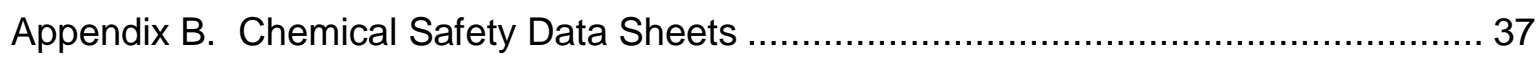




\section{Introduction and Purpose}

This report documents a supplemental analysis to the Hydrotreater/Distillation Column Hazard Analysis Report issued in April 2013. The supplemental analysis session was held March $9^{\text {th }}$ $11^{\text {th }}, 2015$, in response to an independent review comment transmitted to the Pacific Northwest National Laboratory (PNNL) via letter from the U.S. Department of Energy Pacific Northwest Field Office (PNSO; 14-PNSO-0215). The comment states:

“...based on our independent review and in light of our code responsibilities as the owner, we are directing Battelle to provide PNSO with the following items prior to start-up of the distillation column:

"3. Identification of prudent protective actions for the worker and the public (e.g., setback distances, shielding, procedures for minimizing time in the higher risk areas during operation, etc.) based on quantitative analysis and practical risk minimization approaches (for the distillation column).

Items 1 and 3 are also expected to be provided to PNSO for the hydrotreater unit prior to its operation..." (Emphasis added)

The purpose of the review was two-fold: identify prudent, practical protective actions for workers and the public, and use a quantitative analysis as the basis for this selection.

\section{Scope and Methodology}

The baseline analysis upon which this supplemental analysis builds is the Hydrotreater/Distillation Column Hazard Analysis Report (PNNL, 2013) and uses the hazardous conditions cited therein (Table 3-4 of PNNL, 2013) as the basis for evaluation. The hazardous conditions considered were then screened and grouped as follows:

- Items retained included process safety-related hazards that fall outside of the normal operating envelope of PNNL. Standard laboratory hazards, such as trips, slips, falls, work with compressed gases, etc., are within the PNNL normal operating envelope for which safety programs have been implemented and require no further analysis.

- Items retained included those related to operation of the hydrotreater or to utility systems that affect or could be affected by hydrotreater operation. [Items related to operation of the distillation column were evaluated during a supplementary hazard analysis session conducted in June 2014 (PNNL, 2014).] Items related to utility systems that were evaluated in the distillation column report, and for which the hazardous event did not significantly change for hydrotreater operation, were not revisited (i.e., U-30 and U-33).

- Retained items were grouped so that bounding, representative conditions are evaluated. This includes the consideration of initiators, hazard controls, and consequence types. 
This screening and grouping approach provided a subset of hazardous conditions to be analyzed, while assuring that any additional hazard controls selected are broadly applicable to the hazards associated with hydrotreater operations.

The methodology used follows a quantitative approach. The frequency of the initiating event, together with the likelihood that hazard controls (including critical controls) will not function as designed is quantitatively estimated. Failure of all controls is required for the hazardous condition (consequence) to occur. These likelihoods are binned by frequency ranges. The consequences of the hazardous conditions evaluated are also estimated, and binned by severity. Together, the frequency and consequence can be represented for each group of analyzed events in a risk matrix (Figure 1), based on Guidelines for Hazard Evaluation Procedures, $3^{\text {rd }}$ edition (AIChE, 2008).

The risk matrix reflects criteria for risk acceptance that have been adopted for the project and used for the Supplemental Hazard Analysis and Risk Assessment - Distillation Column (PNNL, 2014). A general criterion for a lower level of concern for the most serious accidents may be adopted from various sources. From the U.S. Department of Energy, EH-33 (DOE, 1996), the threshold for significant risk as adopted by the Occupational Safety and Health Administration (OSHA) in its final benzene rule is $10^{-3}$ fatality/year; the average annual accidental fatality risk in U.S. industries is $10^{-4}$ fatality/year. For the public, $10^{-4}$ fatality/year is often used as a maximum tolerable individual risk criterion (Lewis ${ }^{1}$, Travis $^{2}$ ).

For this evaluation, PNNL has established a lower frequency value of $10^{-5}$ hazardous conditions/year for determining risk, which includes the potential for serious accidents affecting workers or the public. This frequency corresponds to the generic catastrophic failure rate for pressure vessels, including those routinely encountered by the public, posing pressure hazards and containing hazardous and flammable materials (Lees, 2012). Should the frequency of a specific hazardous condition fall to a small fraction of the likelihood of a catastrophic pressure vessel failure $\left(10^{-5} /\right.$ year $)$, the risk is considered small enough that additional controls are unnecessary.

When additional controls needed to lower the likelihood of a hazardous condition were considered neither practical nor prudent, actions to reduce consequences were identified instead. This approach follows a control hierarchy consistent with established DOE guidance (DOE, 2006) that emphasizes preventive over mitigative controls, and engineered controls over administrative controls.

For events that do not result in an energetic release from a pressure vessel failure, consequences are less severe and a higher likelihood of occurrence may be justified, based on risk (see Figure 1).

\footnotetext{
${ }^{1}$ Lewis, Steven. "Risk Criteria - When is low enough good enough?" American Society of Safety Engineers- Middle East Chapter. $8^{\text {th }}$ Professional Development Conference \& Exhibition (PDC\&E), February 2008.

2 Travis, C.C., et al., "Cancer risk management: A review of 132 federal regulatory decisions," Environ. Sci. Technol., Vol. 21. No. 5. 1987.
} 
The frequency (f) ranges are defined as follows (per year):

$$
\begin{array}{ll}
1 & 1 \geq f>1 E-01 \\
2 & 1 E-01 \geq f>1 E-03 \\
3 & 1 E-03 \geq f>1 E-05 \\
4 & f \leq 1 E-05
\end{array}
$$

The consequence bins are defined as follows:

1 worker injury not expected

2 worker injury possible

3 worker injury probable; public impact not expected

4 multiple worker injuries possible; public impact possible (transient public)

The combination of frequency and consequence results in designated risk bins, defined as follows (from AIChE, 2008):

I. Unacceptable; should be mitigated with engineering and/or administrative controls to a risk ranking of III or less within 6 months $^{3}$

II. Undesirable; should be mitigated with engineering and/or administrative controls to a risk ranking of III or less within 12 months $^{3}$

III. Acceptable with controls; should be verified that procedures or controls are in place

IV. Acceptable as is; no mitigation required

Figure 1. Risk Matrix
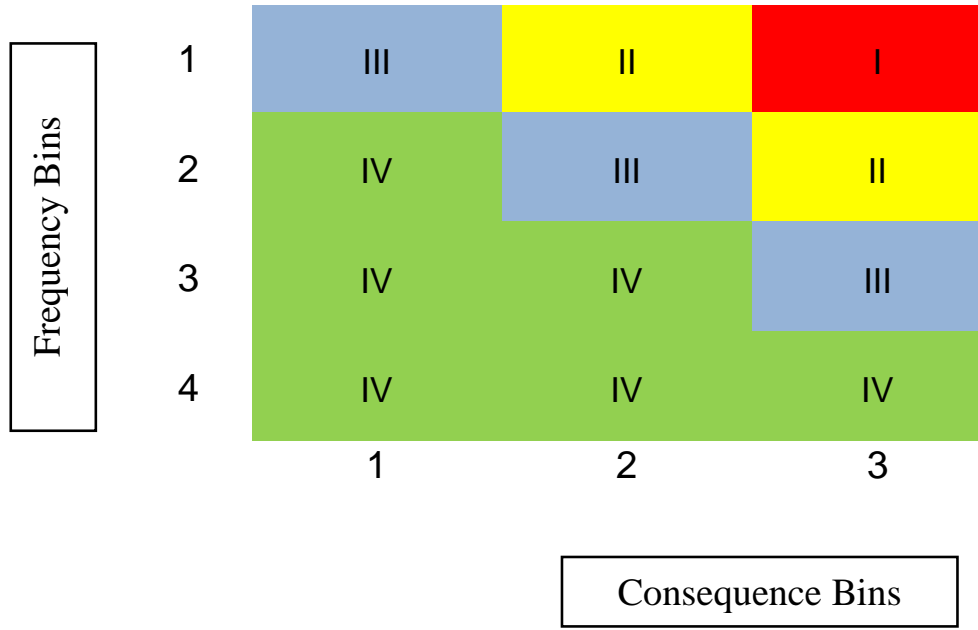

It is noted that the quantitative approach has embedded conservatisms for this application:

- While frequencies of initiating events and control failures are typically based on continuous operation of a process over the course of a year, the intermittent operation

\footnotetext{
${ }^{3}$ The assignation of "unacceptable" and "undesirable" risk bins are essentially the same for the hydrotreater. Controls to reduce the risk ranking to III or less are required to be in place prior to operation.
} 
of the hydrotreater is expected to occur over a small fraction of a full year, thereby reducing risk up to an order of magnitude from that reported.

- Each hazardous condition evaluated does not necessarily include all hazard controls that are in place. Because the events selected are representative, only those with a common set of controls are considered in the analysis.

\section{Results}

The supplemental analysis demonstrates that the hazardous conditions evaluated generally fall into Risk Bin IV with hazard controls in place. Table 2 summarizes the results of the supplemental analysis. The event severities were estimated from the available energy that may be released and environmental conditions postulated to exist for each scenario. The basis for frequency estimates are provided in Table $1^{4}$. Frequencies of failures for which little or no relevant data exists are based on reasonably conservative engineering judgment or follow an accepted convention. For example, the frequency of an administrative control failure is generally considered to be an "anticipated event" (DOE, 2007) and is therefore estimated as 1E01/year in this analysis.

The following additional hazard controls were identified during the analysis as prudent, practical measures to enhance worker safety:

1. Modification of the safe operating procedure (SOP) to state that 1) any leak testing of the system (with nitrogen) will be conducted with the ventilation system operational and 2) the nitrogen system will be closed when not in use. This mitigates the presence of an oxygen deficient atmosphere during maintenance activities and non-operational timeframes. [Action Completed as part of Distillation Column Supplemental Hazard Analysis and Risk Assessment].

2. Modification of the SOP to require the operator to minimize the stay time within the enclosure during hydrotreater operations. This decreases the time an operator is subject to any incidents that might occur within the enclosure. [Action Completed as part of Distillation Column Supplemental Hazard Analysis and Risk Assessment].

3. Administrative controls to check relief header - Modify the SOP to require verification that the low pressure relief line is free of obstruction (e.g., mud dauber nest) (U-7 bounding event).

Two hazardous events, included and noted in Table 2, were prevented due to either the design of gas fittings or to a hydrotreater design change. Specifically, 1) the introduction of oxygen and hydrogen at the same time (H.1-17) was prevented due to compressed oxygen and air having a different fitting connection than compressed nitrogen and 2) an operator inadvertently releasing contents of reactor into the enclosure was prevented with the addition of a 3-way valve (H.6-1).

In addition, questions were raised during the session, regarding critical characteristics of high/low pressure interface valves (H.1-11, H.1-21, H.4-23 and H.4-24) associated with

\footnotetext{
${ }^{4}$ Note that some initiating event frequencies have been adjusted since the April 2013 hazard analysis, based on re-evaluation of the hazardous condition or on the addition of engineered features to the design or administrative controls to procedures.
} 
hazardous events, which were addressed with the aid of additional calculations (Appendix A). Calculations demonstrate that the valves have been appropriately sized for potential flow rates associated with hazardous events and identified controls are sufficient.

For several hazardous events involving leaks of bio-oil, sulfiding agents, and water byproduct, the consequence likelihood would be further reduced from reported levels with the application of required personal protective equipment (Appendix B. Chemical Safety Data Sheets).

Based on the supplemental hazard analysis and the application of the existing and additional hazard controls, the frequencies and mitigated consequences of the potential hazardous conditions were generally considered to fall into Risk Bin IV.

The following additional follow-on action items were identified during the analysis to verify assumptions or enhance worker safety:

1. During startup testing, ensure that valves fail in the appropriate direction, based on their credited function, on loss of air.

2. Review areas of operator proximity to high pressure/high temperature system components for use of personal protective equipment (PPE) against flashing steam release events.

\section{Conclusion}

This supplementary analysis and the quantitative risk evaluation performed has demonstrated that an adequate set of hazard controls are in place to address the potential hazardous conditions associated with hydrotreater operation. Frequencies of initiating events and enabling process control failures were established and considered in evaluating the adequacy of the controls in place.

Additional controls were identified where the quantitative analysis or qualitative considerations (e.g., defense in depth) demonstrated a need for additional preventative or mitigative measures. These include: 1 ) requiring leak testing of the system (with nitrogen) to be conducted with the ventilation system operational and closing the nitrogen system when not in use, 2) limiting stay time for operators within the enclosure, and 3) requiring the verification of an open flow path (with nitrogen) to the relief header prior to each run. These additional controls are considered practical and prudent for further reducing risk to workers and the public, and will be established prior to operation of the hydrotreater unit. In addition, there were follow-on action items identified to verify assumptions or enhance worker safety, including, 1) ensure valves fail in the appropriate direction upon loss of air and 2) evaluate use of PPE for protection against steam release events. 


\section{References}

AIChE, 2008. Guidelines for Hazard Evaluation Procedures, $3^{\text {rd }}$ edition. Center for Chemical Process Safety, New York, New York.

DOE, 2007. DOE-STD-5506-2007, Preparation of Safety Basis Documents for Transuranic (TRU) Waste Facilities. U.S. Department of Energy, Washington, D.C.

DOE, 2006. DOE-STD-3009-94, Change Notice 3, Preparation Guide for U.S. Department of Energy Nonreactor Nuclear Facility Documented Safety Analyses. U.S. Department of Energy, Washington, D.C.

DOE, 1996. Hazard and Barrier Analysis Guidance Document, EH-33 Office of Operating Experience Analysis and Feedback, U.S. Department of Energy, Washington, D.C.

Lees, 2012. Loss Prevention in the Process Industries, 4th Edition. Elsevier, Inc., Oxford, UK.

PNNL, 2013. Hydrotreater/Distillation Column Hazard Analysis Report, April 2013. Pacific Northwest National Laboratory, Richland, Washington.

PNNL, 2014. Supplemental Hazard Analysis and Risk Assessment - Distillation Column, July 2014. Pacific Northwest National Laboratory, Richland, Washington. 
Supplemental Hazard Analysis and Risk Assessment - Hydrotreater

This page intentionally left blank 
Table 1. Frequency Values and Data Sources

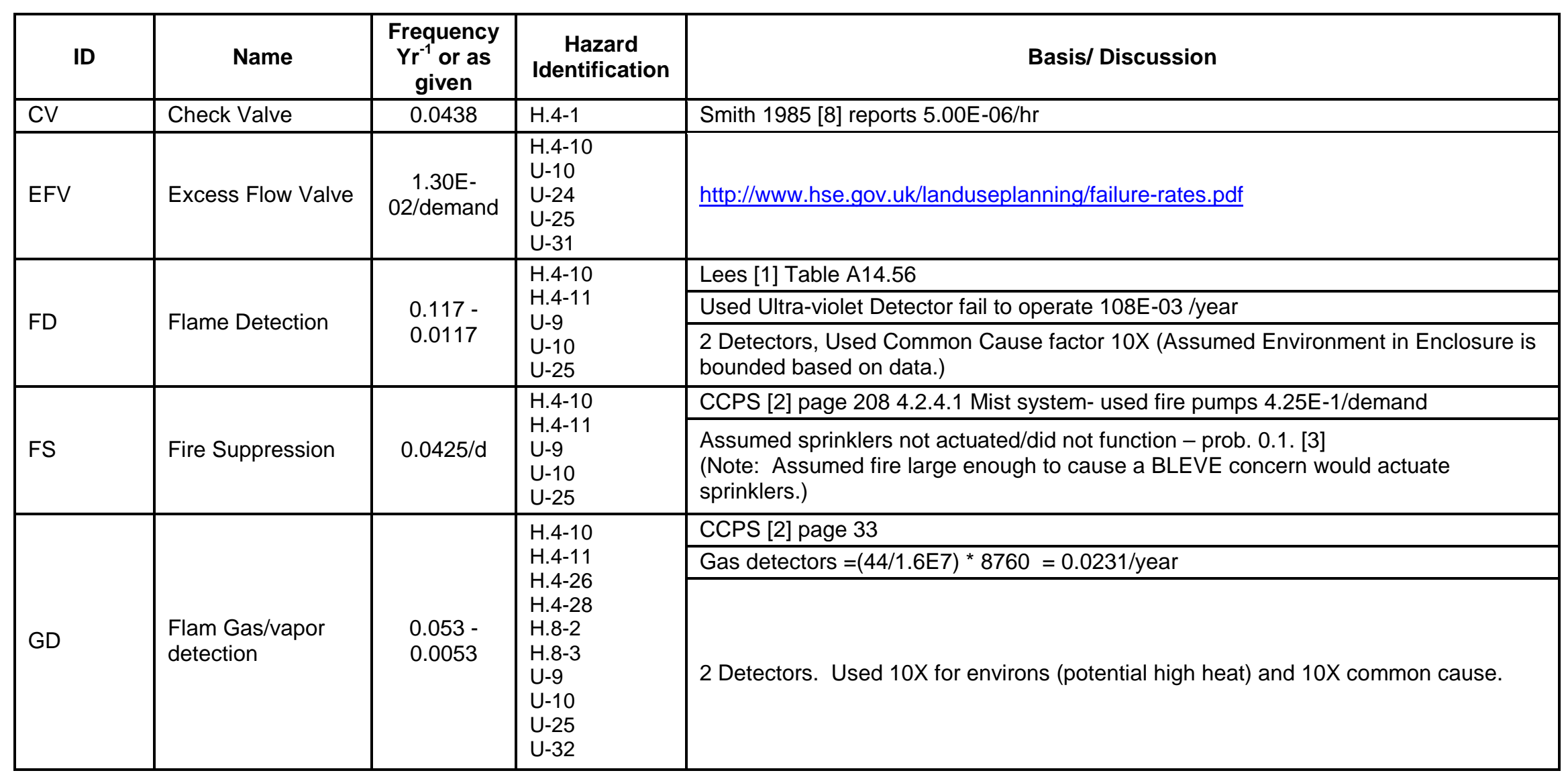


Supplemental Hazard Analysis and Risk Assessment - Hydrotreater

\begin{tabular}{|c|c|c|c|c|}
\hline ID & Name & $\begin{array}{l}\text { Frequency } \\
\text { Yr }^{-1} \text { or as } \\
\text { given }\end{array}$ & $\begin{array}{l}\text { Hazard } \\
\text { Identification }\end{array}$ & Basis/ Discussion \\
\hline IGN1(EN) & $\begin{array}{l}\text { Ignition of spill - } \\
\text { product }\end{array}$ & $\begin{array}{l}0.01- \\
0.001\end{array}$ & $\begin{array}{l}\text { H.1-8 } \\
\text { H.1-12 } \\
\text { H.1-14 } \\
\text { H.2-9 } \\
\text { H.2-17 } \\
\text { H.4-11 } \\
\text { H.5-2 } \\
\text { H.7-5 } \\
\text { H.7-9 } \\
\text { U-3 } \\
\text { U-4 } \\
\text { U-5 } \\
\text { U-6 } \\
\text { U-9 } \\
\text { U-32 }\end{array}$ & $\begin{array}{l}\text { Engineering Assumption: Ignition Range based on spray of "finished product" } \\
(0.01) \text { versus spill (0.001). Assume ignition is } 0.1 \text { levent if outside of Class } 1 \text { Div } 2 \\
\text { Environment. }\end{array}$ \\
\hline IGN2(EN) & 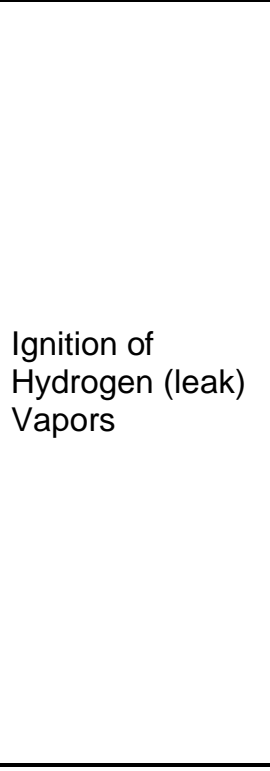 & 5.00E-01 & $\begin{array}{l}\text { H.2-1 } \\
\text { H.2-2 } \\
\text { H.2-3 } \\
\text { H.2-15 } \\
\text { H.4-8 } \\
\text { H.4-10 } \\
\text { H.4-15 } \\
\text { H.4-19 } \\
\text { H. } 4-20 \\
\text { H. } 4-23 \\
\text { H. } 4-24 \\
\text { H. } 4-26 \\
\text { H. } 4-28 \\
\text { H.7-7 } \\
\text { H.7-8 } \\
\text { H.8-2 } \\
\text { H.8-3 } \\
\text { U-10 } \\
\text { U-24 } \\
\text { U-25 } \\
\text { U-31 } \\
\end{array}$ & $\begin{array}{l}\text { Engineering Assumption: } 0.5 \text { for "autoignition" of hydrogen in Class } 1 \text { Div. } 2 \\
\text { Environment. }\end{array}$ \\
\hline IGN3 & Ignition in vessel & 1.00E-03 & U-8 & $\begin{array}{l}\text { Engineering Assumption: Ignition source in vessel. Assumed to be equal to lower } \\
\text { range of IGN1. }\end{array}$ \\
\hline
\end{tabular}




\begin{tabular}{|c|c|c|c|c|}
\hline ID & Name & $\begin{array}{l}\text { Frequency } \\
\mathrm{Yr}^{-1} \text { or as } \\
\text { given }\end{array}$ & $\begin{array}{l}\text { Hazard } \\
\text { Identification }\end{array}$ & Basis/ Discussion \\
\hline LK1 & Leak1 & 1.00E-01 & $\begin{array}{l}\text { H.1-8 } \\
\text { H.1-12 } \\
\text { H.1-14 } \\
\text { H.2-1 } \\
\text { H.2-2 } \\
\text { H.2-3 } \\
\text { H.2-9 } \\
\text { U-3 } \\
\text { U-4 } \\
\text { U-5 } \\
\text { U-6 }\end{array}$ & $\begin{array}{l}\text { Small leak - sufficient to release gas. Little data appears on the use of "Tubing" as } \\
\text { process piping. Failure rates are therefor based on Lees [1] Table A14.8 "Gasket } \\
\text { failure" 1E-06/hour * 10x multiplier to address "small leaks" which appear under } \\
\text { reported 0.087 /year - use 0.1 /year. (Note: Gasket Failures appear in range from } \\
\text { 1E-04/hour to 1E-07/hour - (Serious Leak post-accident Table A14.7) }\end{array}$ \\
\hline LK2 & Leak2 & 1.00E-02 & $\begin{array}{l}\text { H.2-15 } \\
\text { H.3-5 } \\
\text { H. } 4-8 \\
\text { H. } 4-15 \\
\text { H. } 4-19 \\
\text { H. }-20 \\
\text { H.7-8 } \\
\text { H.7-7 } \\
\text { H. } 7-8\end{array}$ & $\begin{array}{l}\text { Moderate Leak (sufficient to result in significant loss of fluid or gas). See above } \\
\text { without 10x multiplier. }\end{array}$ \\
\hline LPR & $\begin{array}{l}\text { Low Pressure } \\
\text { Relief Header } \\
\text { Block }\end{array}$ & 1.00E-02 & & $\begin{array}{l}\text { Engineering Assumption: Based on design including screens and } 2 \text { relief outlets "T" } \\
\text { points. Area is exposed not covered. }\end{array}$ \\
\hline OP1 & $\begin{array}{l}\text { Operator Error } \\
\text { (procedural step or } \\
\text { response to } \\
\text { process alarm, or } \\
\text { readily detectable } \\
\text { event) }\end{array}$ & 1.00E-01 & $\begin{array}{l}\text { H. } 4-8 \\
\text { H. } 4-15 \\
\text { H. } 4-19 \\
\text { H. } 4-20 \\
\text { H. } 7-8\end{array}$ & $\begin{array}{l}\text { (DOE-STD-3009, DOE-STD-5506. High point of Anticipated Event Range. } \\
\text { Anticipated range is recommended unless a rationale for supporting lower frequencies } \\
\text { is provided (e.g., requires multiple independent errors of commission or omission, } \\
\text { activity is rarely performed, etc.) }\end{array}$ \\
\hline
\end{tabular}




\begin{tabular}{|c|c|c|c|c|}
\hline ID & Name & $\begin{array}{l}\text { Frequency } \\
\text { Yr }^{-1} \text { or as } \\
\text { given }\end{array}$ & $\begin{array}{l}\text { Hazard } \\
\text { Identification }\end{array}$ & Basis/ Discussion \\
\hline OP2 & 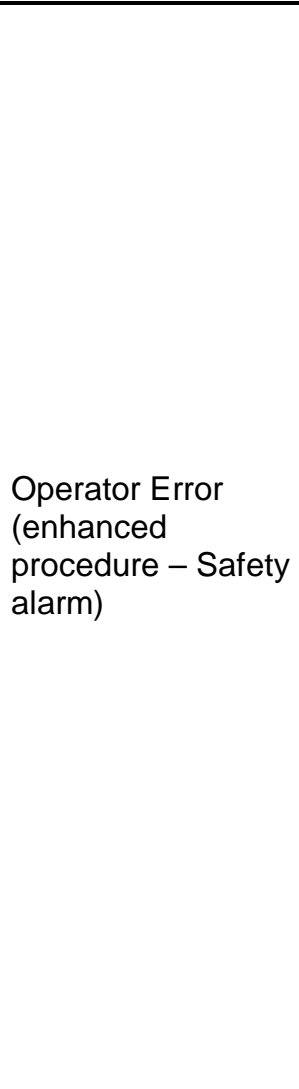 & 5.00E-02 & 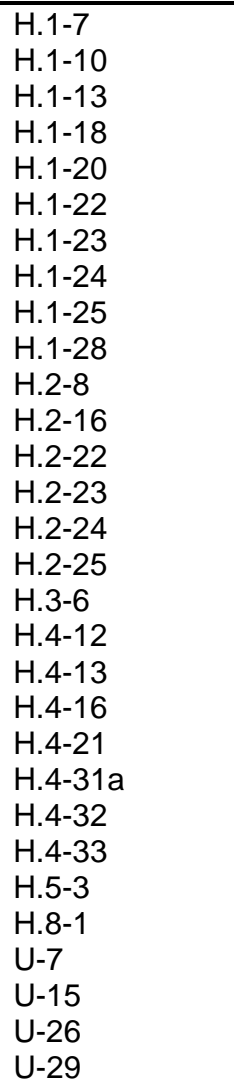 & $\begin{array}{l}\text { EH-33[4] } 5 X \text { Nominal value for failure to respond to Compelling Signal given many } \\
\text { competing signals. }\end{array}$ \\
\hline OP3 & $\begin{array}{l}\text { Operator Error } \\
\text { failure to detect } \\
\text { and respond to off- } \\
\text { normal system }\end{array}$ & $5.00 \mathrm{E}-01$ & $\begin{array}{l}\text { H. } 4-10 \\
\text { H. } 4-11 \\
\text { U-9 } \\
\text { U-10 } \\
\text { U-25 }\end{array}$ & $\begin{array}{l}\text { CCPS [5] Table } 3.17 \text { failure to perform required action (based on contact with control } \\
\text { room) used when event includes a time urgent/higher stress response and for non- } \\
\text { procedure driven operator response actions. }\end{array}$ \\
\hline P1 & $\begin{array}{l}\text { Single Process } \\
\text { Instrument/Alarm }\end{array}$ & 3.00E-01 & $\begin{array}{l}\text { H. } 4-4 \\
\text { H. } 4-5\end{array}$ & $\begin{array}{l}\text { EH-33[4] Table A.1 General Instrumentation (sum of fail to operate and shift } \\
\text { calibration. 3.1E-5/hour) } \\
\text { Lees }[1] \text { Table A14.53. Bounds average of all reported mean failure rates. [(26 } \\
+33+18+56) / 4] / 106 \text { hour }\end{array}$ \\
\hline
\end{tabular}


Supplemental Hazard Analysis and Risk Assessment - Hydrotreater

\begin{tabular}{|c|c|c|c|c|}
\hline ID & Name & $\begin{array}{l}\text { Frequency } \\
\mathrm{Yr}^{-1} \text { or as } \\
\text { qiven }\end{array}$ & $\begin{array}{c}\text { Hazard } \\
\text { Identification }\end{array}$ & Basis/ Discussion \\
\hline P2 & $\begin{array}{l} \\
\\
2 \text { Process } \\
\text { Instruments/ } \\
\text { Alarms }\end{array}$ & 6.00E-02 & $\begin{array}{l}\text { H.1-1 } \\
\text { H.1-2 } \\
\text { H.1-3 } \\
\text { H.1-4 } \\
\text { H.1-5 } \\
\text { H.1-6 } \\
\text { H.1-19 } \\
\text { H.1-27 } \\
\text { H.2-15 } \\
\text { H.2-20 } \\
\text { H.3-2 } \\
\text { H.3-3 } \\
\text { H.3-4 } \\
\text { H.3-6 } \\
\text { H.4-2 } \\
\text { H.4-3 } \\
\text { H.4-6 } \\
\text { H.4-7 } \\
\text { H.4-17 } \\
\text { H.4-27 } \\
\text { H.7-1 } \\
\text { H.7-7 } \\
\text { U-1 } \\
\text { U-2 } \\
\text { U-21 }\end{array}$ & $\begin{array}{l}\text { CCPS [5] PP } 363 \text { Beta Factor } 0.2 \text { (Range 0.1-0.2) For a } 2 \text { component system }=\left(C^{*} F^{\star}\right. \\
\text { Failure Rate) }=0.2^{\star} P 1 .\end{array}$ \\
\hline
\end{tabular}




\begin{tabular}{|c|c|c|c|c|}
\hline ID & Name & $\begin{array}{l}\text { Frequency } \\
\mathrm{Yr}^{-1} \text { or as } \\
\text { given }\end{array}$ & $\begin{array}{c}\text { Hazard } \\
\text { Identification }\end{array}$ & Basis/ Discussion \\
\hline P3 & $\begin{array}{l}\text { Process Critical } \\
\text { Control }\end{array}$ & 1.00E-02 & $\begin{array}{l}\text { H.1-1 } \\
\text { H.1-2 } \\
\text { H.1-3 } \\
\text { H.1-4 } \\
\text { H.1-5 } \\
\text { H.1-6 } \\
\text { H.1-19 } \\
\text { H.1-27 } \\
\text { H.2-20 } \\
\text { H.3-6 } \\
\text { H.4-2 } \\
\text { H.4-3 } \\
\text { H.4-6 } \\
\text { H.4-7 } \\
\text { H.7-1 } \\
\text { U-3 } \\
\text { U-4 } \\
\text { U-5 } \\
\text { U-6 } \\
\text { U-15 }\end{array}$ & $\begin{array}{l}\text { Engineering Assumption: Used Lower End of SIL-1 for Critical Control } \\
\text { Instrumentation [6]. }\end{array}$ \\
\hline PCV & $\begin{array}{l}\text { Pressure Control } \\
\text { Valve }\end{array}$ & 0.0314 & $\begin{array}{l}\text { H.1-15 } \\
\text { H.1-16 } \\
\text { H.2-7 } \\
\text { H.2-10 } \\
\text { H.2-26 } \\
\text { H. } 4-23 \\
\text { H. } 4-24 \\
\text { H. } 4-29 \\
\text { H. } 4-31 B \\
\text { H. } 7-4\end{array}$ & CCPS [2] page 201. 3.5.3.3 Pneumatic operated valves. Mean 3.59E-06/hour \\
\hline PDL & $\begin{array}{l}\text { PDL maintain } \\
\text { Concentration } \\
<\text { limit }\end{array}$ & $\begin{array}{l}0.1 / \\
\text { demand }\end{array}$ & U-24 & $\begin{array}{l}\text { Engineering Assumption: building size/ventilation unable to maintain gas to below } \\
\text { hazardous limits or } \mathrm{LFL}=0.1 / \text { demand }\end{array}$ \\
\hline
\end{tabular}


Supplemental Hazard Analysis and Risk Assessment - Hydrotreater

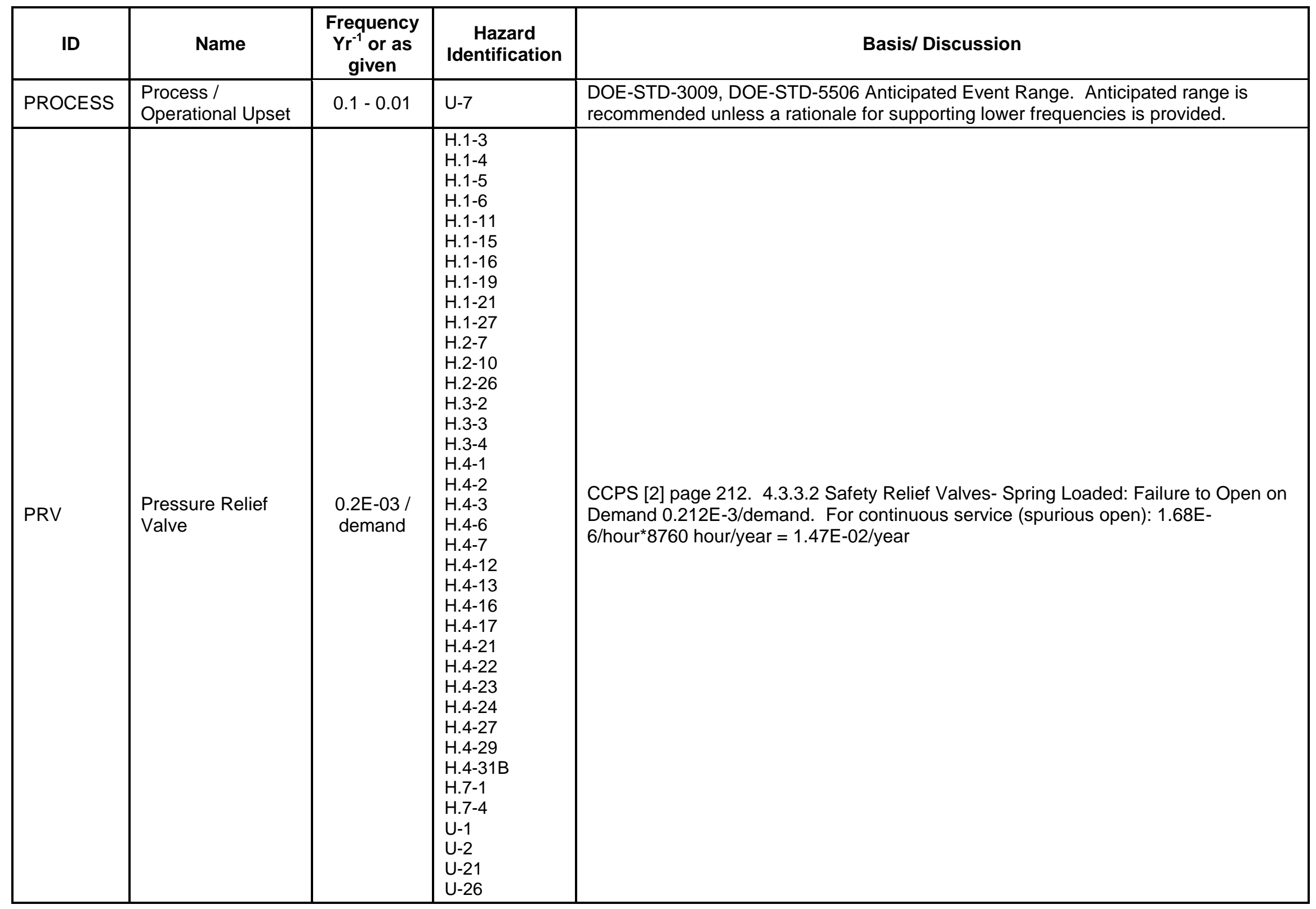


Supplemental Hazard Analysis and Risk Assessment - Hydrotreater

\begin{tabular}{|c|c|c|c|c|}
\hline ID & Name & $\begin{array}{c}\text { Frequency } \\
\mathrm{Yr}^{-1} \text { or as } \\
\text { given }\end{array}$ & $\begin{array}{l}\text { Hazard } \\
\text { Identification }\end{array}$ & Basis/ Discussion \\
\hline PSV & $\begin{array}{l}\text { Pressure Safety } \\
\text { Valve }\end{array}$ & $\begin{array}{l}0.0415 / \\
\text { demand }\end{array}$ & & $\begin{array}{l}\text { CCPS [2] page 211. 4.3.3.2 Safety Relief Valves- Pilot Operated PRV 4.15E-3/d * } 10 \\
\text { for liquid service conditions. }\end{array}$ \\
\hline VC1 & $\begin{array}{l}\text { Catastrophic } \\
\text { Failure of Vessel }\end{array}$ & 1.00E-05 & $\begin{array}{l}\text { H.1-18 } \\
\text { H. } 4-9 \\
\text { H. } 4-18\end{array}$ & $\begin{array}{l}\text { Lees [1] pp } 609 \text { establishes a mean failure rate of 1E-5 for disruptive failures of } \\
\text { pressure vessel (Hurst, Davies et al, 1994). } \\
\text { CCPS [2] 3.6.2.1 Metallic Pressurized Vessel establishes a range 3.85E-4 (upper), } \\
\text { 9.55E-05 (mean) 1.24E-6 (lower) for "all failures". } \\
\text { For Hydrotreater/Distillation Column vessels, the use of 1E-5 failure / year represents } \\
\text { a conservative estimate based on the following considerations: } \\
\text { 1) Use of } 316 \text { Stainless Steel (SS) as material of construction, SS provides excellent } \\
\text { resistance to corrosion and a number of material degradations issues for the } \\
\text { conditions experience in HT/DC operations; SS ductility provides additional protection } \\
\text { against catastrophic failures associated with (PVB and BLEVEs); the high ultimate } \\
\text { strength SS provides an additional factor of safety compared to carbon steels } \\
\text { commonly used in pressure vessels and thus included the above aggregate values. } \\
\text { 2) Large margin between design pressure and normal operating pressure; } \\
\text { 3) Demonstrated protection systems and environment minimize challenges } \\
\text { (temperature, pressure, environment) to the vessels } \\
\text { 4) Numerous sources (e.g., EH-33 [4]. Pittiglio [7]) identify "high standard" vessels } \\
\text { having a factor of } 10 \text { lower failure frequency - it is assumed high standard equates to } \\
\text { ASME standard or equivalent with modern construction techniques (post-1990) when } \\
\text { looking at "world-wide" population of pressure vessels. }\end{array}$ \\
\hline
\end{tabular}




\begin{tabular}{|c|c|c|c|c|}
\hline ID & Name & $\begin{array}{l}\text { Frequency } \\
\mathrm{Yr}^{-1} \text { or as } \\
\text { given }\end{array}$ & $\begin{array}{c}\text { Hazard } \\
\text { Identification }\end{array}$ & Basis/ Discussion \\
\hline VENT & Ventilation & 1.00E-03 & $\begin{array}{l}\text { H.1-7 } \\
\text { H.1-8 } \\
\text { H.1-10 } \\
\text { H.1-12 } \\
\text { H.1-14 } \\
\text { H.1-20 } \\
\text { H.2-1 } \\
\text { H.2-2 } \\
\text { H.2-3 } \\
\text { H.2-9 } \\
\text { H.2-24 } \\
\text { H.2-25 } \\
\text { H.4-4 } \\
\text { H.4-5 } \\
\text { H.4-8 } \\
\text { H.4-15 } \\
\text { H. } 4-19 \\
\text { H.4-20 } \\
\text { H.4-26 } \\
\text { H.4-28 } \\
\text { H.4-33 } \\
\text { H.5-3 } \\
\text { H.7-8 } \\
\text { H.8-1 } \\
\text { H.8-2 } \\
\text { H.8-3 } \\
\text { U.29 }\end{array}$ & $\begin{array}{l}\text { Engineering Assumption: Assumed failure per demand (Leaks) for which the } \\
\text { ventilation system is not capable of maintaining air concentration in acceptable range. } \\
\text { Assumes system is operation when process is running. }\end{array}$ \\
\hline
\end{tabular}

[1] F. P. Lees, Loss Prevention in the Process Industries, $4^{\text {th }}$ Edition, 2012.

[2] CCPS, Guidelines for Process Equipment Reliability Data, 1989.

[3] NFPA, "U.S. Experience with Sprinklers," John R. Hall, Jr. June 2013.

[4] DOE EH-33, HAZARD AND BARRIER ANALYSIS GUIDANCE DOCUMENT, 1996.

[5] CCPS, Guidelines for Chemical Process Quantitative Risk Analysis, 2nd Edition.

[6] ANSI/ISA 84, Application of Safety Instrumented Systems for the Process Industries, 2004.

[7] Pittiglio, P. et al. "Updated failure rates and risk management in process industries," $68^{\text {th }}$ Conference of the Italian Thermal Machines Engineering Association, ATI2013.

[8] Smith, D.J., Reliability and Maintainability in Perspective, 2nd and 3rd editions, Macmillan London, 1985. 
Supplemental Hazard Analysis and Risk Assessment - Hydrotreater

This page intentionally left blank 


\section{Table 2. Supplemental Hazard Analysis and Risk Assessment Evaluation - Hydrotreater}

\begin{tabular}{|c|c|c|c|c|c|c|c|c|c|}
\hline Hazardous Event & $\begin{array}{l}\text { Representative } \\
\text { Events }\end{array}$ & $\begin{array}{c}\text { Hazard Controls } \\
\text { Bold }=\text { new selected control } \\
\text { Italics = existing control newly } \\
\text { assigned }\end{array}$ & $\begin{array}{l}\text { Initiating } \\
\text { Event } \\
\text { Frequency }\end{array}$ & $\begin{array}{l}\text { (Enabling) } \\
\text { Process } \\
\text { Control } \\
\text { Failures }\end{array}$ & $\begin{array}{l}\text { Critical } \\
\text { Controls }\end{array}$ & $\begin{array}{c}\text { Event } \\
\text { Frequency }\end{array}$ & $\begin{array}{c}\text { Consequence } \\
\text { Severity }\end{array}$ & $\begin{array}{l}\text { Risk } \\
\text { Bin }\end{array}$ & Comments \\
\hline $\begin{array}{l}\text { Catastrophic } \\
\text { failure of high } \\
\text { pressure/high } \\
\text { temperature } \\
\text { vessel due to } \\
\text { material failure } \\
\text { (BLEVE) }\end{array}$ & $\begin{array}{l}\text { H. } 4-9 \\
\text { H.4-18 }\end{array}$ & $\begin{array}{l}\text { R-130 Design (VC1) } \\
\text { Procedural steps: Pressure test } \\
\text { procedure and vessel inspection } \\
\text { Operating within design } \\
\text { parameters } \\
\text { Design of V-140/LG-142 psi } \\
\text { MAWP (VC1) } \\
\text { Level control on V-140 } \\
\text { Pressure/temperature indicators } \\
\text { and alarms }\end{array}$ & 1.00E-05 & & & $1.00 \mathrm{E}-05$ & 4 & IV & $\begin{array}{l}\text { IE due to } \\
\text { catastrophic failure } \\
\text { of vessel material } \\
\text { (VC1). } \\
\text { Pressure checks/ } \\
\text { inspection validate } \\
\text { assumed failure } \\
\text { rate }\end{array}$ \\
\hline $\begin{array}{l}\text { Catastrophic } \\
\text { failure of vessel } \\
\text { due to } \\
\text { overheating } \\
\text { (BLEVE) }\end{array}$ & $\begin{array}{l}\text { H.1-1 } \\
\text { H.1-2 } \\
\text { H.2-20 }\end{array}$ & $\begin{array}{l}\text { Procedural steps: lift procedure } \\
\text { and pressure test } \\
\text { Heater circuits are fused } \\
\text { (protection against short circuit; } \\
\text { P2). } \\
\text { Pressure indication (alarm) on } \\
\text { furnace Z purge. } \\
\text { Software prevents operator from } \\
\text { changing settings above } \\
\text { maximum set point of } 425^{\circ} \mathrm{C} \\
\text { (P2). } \\
\text { There is an internal } \\
\text { thermocouple interlocked to } \\
\text { furnace control (P2). } \\
\text { Cascade furnace control (P2) } \\
\text { R-130 High-High temperature } \\
\text { (P3). } \\
\text { Design of R-130, } 3000 \text { psi } \\
\text { MAWP at } 537^{\circ} \mathrm{C} \text { (shell) }\end{array}$ & 1.00E-01 & $\mathrm{P} 2 * \mathrm{P} 2$ & P3 & $3.60 \mathrm{E}-06$ & 4 & IV & $\begin{array}{l}\text { Use of 2- P2 } \\
\text { reflects multiple } \\
\text { means of } \\
\text { identifying/ } \\
\text { preventing event. }\end{array}$ \\
\hline
\end{tabular}




\begin{tabular}{|c|c|c|c|c|c|c|c|c|c|}
\hline Hazardous Event & $\begin{array}{c}\text { Representative } \\
\text { Events }\end{array}$ & $\begin{array}{c}\text { Hazard Controls } \\
\text { Bold }=\text { new selected control } \\
\text { Italics }=\text { existing control newly } \\
\text { assigned }\end{array}$ & $\begin{array}{l}\text { Initiating } \\
\text { Event } \\
\text { Frequency }\end{array}$ & $\begin{array}{l}\text { (Enabling) } \\
\text { Process } \\
\text { Control } \\
\text { Failures }\end{array}$ & $\begin{array}{l}\text { Critical } \\
\text { Controls }\end{array}$ & $\begin{array}{c}\text { Event } \\
\text { Frequency }\end{array}$ & $\begin{array}{l}\text { Consequence } \\
\text { Severity }\end{array}$ & $\begin{array}{l}\text { Risk } \\
\text { Bin }\end{array}$ & Comments \\
\hline $\begin{array}{l}\text { Operator error- } \\
\text { plug in system } \\
\text { resulting in } \\
\text { catastrophic } \\
\text { failure of vessel } \\
\text { (BLEVE) }\end{array}$ & $\begin{array}{l}\text { H.1-3 } \\
\text { H.1-4 } \\
\text { H.1-5 } \\
\text { H.1-6 } \\
\text { H.1-19 } \\
\text { H.1-27 } \\
\text { H.4-2 } \\
\text { H.4-3 } \\
\text { H.4-6 } \\
\text { H. } 7-1\end{array}$ & $\begin{array}{l}\text { Procedural step - gradual } \\
\text { startup, pressure test, pressure } \\
\text { monitoring and catalyst } \\
\text { monitoring } \\
\text { High pressure nitrogen supply } \\
\text { set @ } 3000 \text { psi. } \\
\text { High pressure nitrogen system } \\
\text { set @ } 2500 \text { psi. } \\
\text { Pre-testing/qualification } \\
\text { (benchtop) of new catalyst - } \\
\text { sulfiding combinations for use } \\
\text { with bio-oils. } \\
\text { Limited to } 1 \text { liter due to design of } \\
\text { pumps. System would slow } \\
\text { down and not get as much. } \\
\text { R-130 Design pressure } 3000 \text { psi. } \\
\text { Pressure relief valve set at } 3000 \\
\text { psi (PRV) } \\
\text { ISCO Pump high pressure set } \\
\text { point (process controlled) (P2). } \\
\text { ISCO pump firmware maximum } \\
\text { pressure of } 3750 \text { psi (P2). } \\
\text { Hydrogen system pressure set } \\
\text { point (3000 psi). } \\
\text { High-High pressure interlock at } \\
2700 \text { psi (P3). } \\
\text { High pressure process control } \\
\text { alarms (P2) }\end{array}$ & $1.00 \mathrm{E}-01$ & $\mathrm{P} 2$ & P3*PRV & $1.20 \mathrm{E}-08$ & 4 & IV & $\begin{array}{l}\text { Pyrophoric } \\
\text { catalysts are not } \\
\text { currently proposed. } \\
\text { Cannot overfill with } \\
\text { catalyst and close } \\
\text { reactor head. } \\
\text { P2 - conservatively } \\
\text { reflects additional } \\
\text { failures required to } \\
\text { challenge pressure } \\
\text { boundary. ISCO } \\
\text { pump versus } \\
\text { failure in H2 or N2 } \\
\text { systems. }\end{array}$ \\
\hline $\begin{array}{l}\text { Catastrophic } \\
\text { failure due to } \\
\text { excessive } \\
\text { exothermic } \\
\text { reaction - feed } \\
\text { rate started too } \\
\text { high (BLEVE) }\end{array}$ & H.3-6 & $\begin{array}{l}\text { Procedural step. } \\
\text { Temperature control (P2) } \\
\text { R-130 High temperature Alarm } \\
\text { on thermocouple (OP2). } \\
\text { High High temperature interlock } \\
\text { (P3) }\end{array}$ & $1.00 \mathrm{E}-01$ & $\mathrm{OP} 2 * \mathrm{P} 2$ & P3 & 3.00E-06 & 4 & IV & $\begin{array}{l}\text { Operator response } \\
\text { to audible alarm } \\
\text { should be to } \\
\text { reduce bio-oil flow. }\end{array}$ \\
\hline
\end{tabular}




\begin{tabular}{|c|c|c|c|c|c|c|c|c|c|}
\hline Hazardous Event & $\begin{array}{c}\text { Representative } \\
\text { Events }\end{array}$ & $\begin{array}{c}\text { Hazard Controls } \\
\text { Bold }=\text { new selected control } \\
\text { Italics }=\text { existing control newly } \\
\text { assigned }\end{array}$ & $\begin{array}{l}\text { Initiating } \\
\text { Event } \\
\text { Frequency }\end{array}$ & $\begin{array}{l}\text { (Enabling) } \\
\text { Process } \\
\text { Control } \\
\text { Failures }\end{array}$ & $\begin{array}{l}\text { Critical } \\
\text { Controls }\end{array}$ & $\begin{array}{c}\text { Event } \\
\text { Frequency }\end{array}$ & $\begin{array}{l}\text { Consequence } \\
\text { Severity }\end{array}$ & $\begin{array}{c}\text { Risk } \\
\text { Bin }\end{array}$ & Comments \\
\hline $\begin{array}{l}\text { Catastrophic } \\
\text { failure due to } \\
\text { excessive } \\
\text { exothermic } \\
\text { reaction } \\
\text { combined with a } \\
\text { plug resulting in } \\
\text { pressure } \\
\text { increase } \\
\text { (BLEVE) }\end{array}$ & H.4-7 & $\begin{array}{l}\text { R-130 design pressure } 3000 \text { psi. } \\
\text { Pressure relief valve set at } 3000 \\
\text { psi (PRV). } \\
\text { Pressure set point on hydrogen } \\
\text { system ( } 3000 \text { psi). } \\
\text { High-High pressure interlock at } \\
2700 \text { psi (P3). } \\
\text { High High temperature interlock } \\
\text { (P3) } \\
\text { ISCO pump high pressure set } \\
\text { point (process controlled) (P2). } \\
\text { ISCO pump firmware maximum } \\
\text { pressure of } 3750 \text { psi (P2). } \\
\text { ISCO pump shear pin at } 4500 \\
\text { psi. } \\
\text { Process control alarms for high } \\
\text { pressure } \\
\text { Procedural step: temperature } \\
\text { feedback } \\
\text { Audible alarms on internal } \\
\text { thermocouple }\end{array}$ & 1.00E-01 & P2 & $P R V * P 3$ & 1.20E-08 & 4 & IV & $\begin{array}{l}\text { Operator response } \\
\text { to audible alarm } \\
\text { should be to turn } \\
\text { off the bio-oil feed } \\
\text { and/or the } \\
\text { hydrogen. }\end{array}$ \\
\hline $\begin{array}{l}\text { Introducing } \\
\text { hydrogen instead } \\
\text { of nitrogen } \\
\text { during pressure } \\
\text { check }\end{array}$ & H.1-18 & $\begin{array}{l}\text { Pressure test procedures } \\
\text { (detection of no pressure on } \\
\text { system components)/ } \\
\text { Valve lineup and labeling (OP2) } \\
\text { Vessel (VC1) }\end{array}$ & $1.00 \mathrm{E}-01$ & OP2 & VC1 & 5.00E-08 & 3 & IV & $\begin{array}{l}\text { Catalyst is ignition } \\
\text { source. Limited } \\
\text { flammability range } \\
\text { (would quickly } \\
\text { purge all O2). } \\
\text { Vessel design } \\
\text { rating higher than } \\
\text { potential pressure } \\
\text { conditions. }\end{array}$ \\
\hline $\begin{array}{l}\text { Introducing } \\
\text { oxygen and } \\
\text { hydrogen at } \\
\text { same time }\end{array}$ & H.1-17 & $\begin{array}{l}\text { Fittings are all different (air, } \\
\text { oxygen, and nitrogen). } \\
\text { Receipt control for oxarc gas } \\
\text { delivery. } \\
\text { Valve and line labeling }\end{array}$ & 1.00E-02 & & $\begin{array}{l}\text { Gas } \\
\text { fittings }\end{array}$ & Prevented & & & \\
\hline
\end{tabular}




\begin{tabular}{|c|c|c|c|c|c|c|c|c|c|}
\hline Hazardous Event & $\begin{array}{c}\text { Representative } \\
\text { Events }\end{array}$ & $\begin{array}{c}\text { Hazard Controls } \\
\text { Bold = new selected control } \\
\text { Italics }=\text { existing control newly } \\
\text { assigned }\end{array}$ & $\begin{array}{l}\text { Initiating } \\
\text { Event } \\
\text { Frequency }\end{array}$ & $\begin{array}{l}\text { (Enabling) } \\
\text { Process } \\
\text { Control } \\
\text { Failures }\end{array}$ & $\begin{array}{l}\text { Critical } \\
\text { Controls }\end{array}$ & $\begin{array}{c}\text { Event } \\
\text { Frequency }\end{array}$ & $\begin{array}{c}\text { Consequence } \\
\text { Severity }\end{array}$ & $\begin{array}{c}\text { Risk } \\
\text { Bin }\end{array}$ & Comments \\
\hline $\begin{array}{l}\text { Catastrophic } \\
\text { failure of reactor } \\
\text { vessel due to } \\
\text { flame } \\
\text { impingement } \\
\text { from hydrogen } \\
\text { leak (BLEVE) }\end{array}$ & $\begin{array}{l}\mathrm{H} .4-10 \\
\mathrm{U}-10 \\
\mathrm{U}-25\end{array}$ & $\begin{array}{l}\text { Design of hydrogen pipe system, } \\
\text { furnace enclosure and } \\
\text { enclosure. } \\
\text { Excess flow valve on hydrogen } \\
\text { supply (EFV). } \\
\text { Flame detection inside enclosure } \\
\text { (FD). } \\
\text { Hydrogen monitor on skid and } \\
\text { enclosure (GD). } \\
\text { Fire suppression system in } \\
\text { enclosure (FS). } \\
\text { Operator response and } \\
\text { emergency stop (OP3). }\end{array}$ & 1.00E-02 & $\begin{array}{l}\text { IGN2(EN)* } \\
\text { OP3 }\end{array}$ & $\begin{array}{l}\text { GD*FD* } \\
F S^{\star} E F V\end{array}$ & 8.57E-09 & 4 & IV & $\begin{array}{l}\text { IE due to ignition of } \\
\text { hydrogen }\end{array}$ \\
\hline $\begin{array}{l}\text { Catastrophic } \\
\text { failure of reactor } \\
\text { vessel due to } \\
\text { flame } \\
\text { impingement } \\
\text { from liquid fire } \\
\text { (BLEVE) }\end{array}$ & $\begin{array}{l}\text { H.4-11 } \\
\text { U-9 }\end{array}$ & $\begin{array}{l}\text { Design of product tank ( } \mathrm{V}-160 \\
\text { A/B) and furnace enclosure. } \\
\text { Pressure test procedure } \\
\text { Distillation skid has separate } \\
\text { containment from hydrotreater } \\
\text { skid } \\
\text { Enclosure design - Class } 1 \\
\text { Division } 2 . \\
\text { Flammable vapor monitor on } \\
\text { skid (GD). } \\
\text { Flame detection inside enclosure } \\
\text { (FD). } \\
\text { Fire suppression system in } \\
\text { enclosure (FS). } \\
\text { Operator response and } \\
\text { emergency stop (OP3). }\end{array}$ & 1.00E-02 & $\begin{array}{l}\text { IGN1(EN)* } \\
\text { OP3 }\end{array}$ & $\begin{array}{l}\text { GD*FD* } \\
F S\end{array}$ & $1.32 \mathrm{E}-08$ & 4 & IV & $\begin{array}{l}\text { Event may be } \\
\text { incredible based on } \\
\text { lack of quantity or } \\
\text { heat energy from } \\
\text { pool fire. } \\
\text { Product tank } \\
\text { V-160 A/B size is } \\
19 L \text { ( } 1 \text { days' } \\
\text { running production } \\
\text { at } 1-2 \text { L/hr }-38 \mathrm{~L} \\
\text { total volume of } \\
\text { both product } \\
\text { tanks). } \\
\text { IE due to } \\
\text { significant product } \\
\text { spill }\end{array}$ \\
\hline
\end{tabular}




\begin{tabular}{|c|c|c|c|c|c|c|c|c|c|}
\hline Hazardous Event & $\begin{array}{c}\text { Representative } \\
\text { Events }\end{array}$ & $\begin{array}{c}\text { Hazard Controls } \\
\text { Bold }=\text { new selected control } \\
\text { Italics }=\text { existing control newly } \\
\text { assigned }\end{array}$ & $\begin{array}{l}\text { Initiating } \\
\text { Event } \\
\text { Frequency }\end{array}$ & $\begin{array}{l}\text { (Enabling) } \\
\text { Process } \\
\text { Control } \\
\text { Failures }\end{array}$ & $\begin{array}{l}\text { Critical } \\
\text { Controls }\end{array}$ & $\begin{array}{c}\text { Event } \\
\text { Frequency }\end{array}$ & $\begin{array}{l}\text { Consequence } \\
\text { Severity }\end{array}$ & $\begin{array}{c}\text { Risk } \\
\text { Bin }\end{array}$ & Comments \\
\hline $\begin{array}{l}\text { Catastrophic } \\
\text { failure of vessel } \\
\text { due to over } \\
\text { pressurization } \\
\text { from high } \\
\text { pressure } \\
\text { nitrogen system } \\
\text { (PVB) }\end{array}$ & H.1-16 & $\begin{array}{l}\text { Pressure control of the high } \\
\text { pressure nitrogen supply system } \\
\text { at } 2500 \text { psi (PCV). } \\
\text { Pressure relief of the high } \\
\text { pressure nitrogen supply to } 3000 \\
\text { psi (PRV). } \\
\text { Pressure control of high } \\
\text { pressure nitrogen system } \\
\text { pressure set at } 2500 \text { psi (PCV). } \\
\text { High pressure component PRVs } \\
\text { set at } 3000 \text { psi (PRV). } \\
\text { High pressure vessel and } \\
\text { component design pressure } \geq \\
3000 \text { psi. } \\
\text { High pressure alarms. } \\
\text { High pressure interlocks. }\end{array}$ & 1.00E-01 & $P R V * P C V$ & PRV & $1.26 \mathrm{E}-10$ & 3 & IV & $\begin{array}{l}\text { IE reflects operator } \\
\text { error in mis-setting } \\
\text { one of } 2 \mathrm{PCVs} \\
\text { (bounds random } \\
\text { failure of PCV. }\end{array}$ \\
\hline $\begin{array}{l}\text { Operator error - } \\
\text { resulting in over } \\
\text { pressurization of } \\
\text { the system } \\
\text { (PVB) }\end{array}$ & H.2-10 & $\begin{array}{l}\text { Design of R-130/V-140 and } \\
\text { component pressure } \geq 3000 \text { psi } \\
\text { MAWP. } \\
\text { R-130/V-140 PRVs set at } 3000 \\
\text { psi (PRV). } \\
\text { Pressure regulation of the } \\
\text { hydrogen supply to } 3000 \text { psi } \\
\text { (PCV). } \\
\text { Pressure regulation of hydrogen } \\
\text { system pressure set at } 2500 \text { psi } \\
\text { (PCV). } \\
\text { High pressure alarm in R-130. } \\
\text { High-High pressure interlock in } \\
\text { R-130. } \\
\text { Procedural step for pressure set } \\
\text { point and verify gas flow. }\end{array}$ & 1.00E-01 & $P R V * P C V$ & PRV & $1.26 \mathrm{E}-10$ & 3 & IV & $\begin{array}{l}\text { IE reflects operator } \\
\text { error in mis-setting } \\
\text { one of } 2 \text { PCVs } \\
\text { (bounds random } \\
\text { failure of PCV. }\end{array}$ \\
\hline
\end{tabular}




\begin{tabular}{|c|c|c|c|c|c|c|c|c|c|}
\hline Hazardous Event & $\begin{array}{c}\text { Representative } \\
\text { Events }\end{array}$ & $\begin{array}{c}\text { Hazard Controls } \\
\text { Bold }=\text { new selected control } \\
\text { Italics }=\text { existing control newly } \\
\text { assigned }\end{array}$ & $\begin{array}{l}\text { Initiating } \\
\text { Event } \\
\text { Frequency }\end{array}$ & $\begin{array}{l}\text { (Enabling) } \\
\text { Process } \\
\text { Control } \\
\text { Failures }\end{array}$ & $\begin{array}{l}\text { Critical } \\
\text { Controls }\end{array}$ & $\begin{array}{c}\text { Event } \\
\text { Frequency }\end{array}$ & $\begin{array}{l}\text { Consequence } \\
\text { Severity }\end{array}$ & $\begin{array}{c}\text { Risk } \\
\text { Bin }\end{array}$ & Comments \\
\hline $\begin{array}{l}\text { Operator error - } \\
\text { failure to } \\
\text { open/close valve } \\
\text { resulting in } \\
\text { catastrophic } \\
\text { failure of vessel } \\
\text { (PVB) }\end{array}$ & $\begin{array}{l}\text { H.1-11 } \\
\text { H.1-21 }\end{array}$ & $\begin{array}{l}\text { Procedural Step (including valve } \\
\text { lineup). } \\
\text { Pressure relief valves on low } \\
\text { pressure system components } \\
\text { (PRV). }\end{array}$ & 2.00E-02 & & PRV & 4.00E-06 & 3 & IV & $\begin{array}{l}\text { Relief valves have } \\
\text { been sized for this } \\
\text { scenario. } \\
\text { IE reduced due to } \\
\text { requirement of two } \\
\text { valves to be } \\
\text { misaligned. }\end{array}$ \\
\hline $\begin{array}{l}\text { Excessive } \\
\text { pressure in low } \\
\text { pressure } \\
\text { nitrogen system } \\
\text { resulting in } \\
\text { catastrophic } \\
\text { failure of the } \\
\text { vessel (PVB) }\end{array}$ & $\begin{array}{l}\text { Н.1-15 } \\
\text { Н.2-7 } \\
\text { H.2-26 } \\
\text { H. } 4-29 \\
\text { H. } 4-31 B \\
\text { H. } 7-4\end{array}$ & $\begin{array}{l}\text { Pressure regulation of the low } \\
\text { pressure nitrogen supply to } 100 \\
\text { psi (PCV) } \\
\text { Pressure regulation of the low } \\
\text { pressure nitrogen system } \\
\text { pressure to } 10 \text { psi (PCV). } \\
\text { Low pressure component PRVs } \\
\text { set at } \leq 100 \text { psi (PRV). } \\
\text { Pressure test procedure } \\
\text { Design of V-160 A/B } 155 \text { psi } \\
\text { V-160 A/B PRV set at } 70 \text { psi. } \\
\text { Containment pan on skid } \\
\text { lenclosure. } \\
\text { Design V-125 } 155 \text { psi MAWP, V- } \\
125 \text { PRV set at } \leq 100 \text { psi (PRV) } \\
\text { Low pressure system pressure } \\
\text { limit. } \\
\text { Pressure set point, system } \\
\text { pressure control. } \\
\text { Enclosure Design - Class } 1 \\
\text { Division } 2 . \\
\text { Enclosure ventilation system } \\
\text { Flammable vapor monitoring on } \\
\text { skid. } \\
\text { Fire suppression system in } \\
\text { enclosure. }\end{array}$ & $1.00 \mathrm{E}-01$ & $P R V * P C V$ & PRV & $1.26 \mathrm{E}-10$ & 3 & IV & $\begin{array}{l}\text { IE reflects operator } \\
\text { error in mis-setting } \\
\text { one of } 2 \text { PCVs } \\
\text { (bounds random } \\
\text { failure of PCV. }\end{array}$ \\
\hline
\end{tabular}




\begin{tabular}{|c|c|c|c|c|c|c|c|c|c|}
\hline Hazardous Event & $\begin{array}{l}\text { Representative } \\
\text { Events }\end{array}$ & $\begin{array}{c}\text { Hazard Controls } \\
\text { Bold }=\text { new selected control } \\
\text { Italics }=\text { existing control newly } \\
\text { assigned }\end{array}$ & $\begin{array}{l}\text { Initiating } \\
\text { Event } \\
\text { Frequency }\end{array}$ & $\begin{array}{l}\text { (Enabling) } \\
\text { Process } \\
\text { Control } \\
\text { Failures }\end{array}$ & $\begin{array}{l}\text { Critical } \\
\text { Controls }\end{array}$ & $\begin{array}{c}\text { Event } \\
\text { Frequency }\end{array}$ & $\begin{array}{c}\text { Consequence } \\
\text { Severity }\end{array}$ & $\begin{array}{l}\text { Risk } \\
\text { Bin }\end{array}$ & Comments \\
\hline $\begin{array}{l}\text { Catastrophic } \\
\text { failure of vessel } \\
\text { due to ISCO } \\
\text { pump failure } \\
\text { results in liquid } \\
\text { backflow (PVB) }\end{array}$ & H.4-1 & $\begin{array}{l}\text { PSE2005 @ } 100 \text { psi sized for } \\
\text { backflow (PRV). } \\
\text { Check valve, CK-2007 and CK- } \\
2012(\mathrm{CV}) \text {. }\end{array}$ & 2.00E-02 & $\mathrm{CV}^{*} \mathrm{CV}$ & PRV & 7.67E-09 & 3 & IV & $\begin{array}{l}\text { IE reduced due to } \\
\text { requirement of } \\
\text { multiple failures in } \\
\text { ISCO pump }\end{array}$ \\
\hline $\begin{array}{l}\text { Catastrophic } \\
\text { failure of vessel } \\
\text { due to valve } \\
\text { failure/ over } \\
\text { pressurization } \\
\text { (PVB) }\end{array}$ & H.4-22 & $\begin{array}{l}\text { PSV-4016 set at } 70 \text { psi (PRV). } \\
\text { LG-147 designed at } 100 \text { psig } \\
\text { MAWP. } \\
\text { LG-147 vented to product gas } \\
\text { header. } \\
\text { PSV-5001 in product gas vent } \\
\text { header downstream of LG-147 is } \\
\text { set at } 10 \text { psi (PRV). }\end{array}$ & 1.00E-01 & & PRV*PRV & 4.00E-09 & 3 & IV & \\
\hline $\begin{array}{l}\text { Catastrophic } \\
\text { failure of vessel } \\
\text { due to a fast } \\
\text { block in flow path } \\
\text { (PVB) } \\
\text { (bounds spray or } \\
\text { spill) }\end{array}$ & $\begin{array}{l}\text { H.3-2 } \\
\text { H.3-3 } \\
\text { H.3-4 } \\
\text { H.4-17 } \\
\text { H.4-27 } \\
\text { U-1 } \\
\text { U-2 } \\
\text { U-21 }\end{array}$ & $\begin{array}{l}\text { Design of V-140 -3000 psi } \\
\text { MAWP. } \\
\text { Pressure relief valve PSV -4002 } \\
\text { set at } 3000 \text { psi (PRV). } \\
\text { PRV in reactor PSV-3011 } \\
\text { (PRV) } \\
\text { Level control on V-140 } \\
\text { (indicator/alarm). Pressure } \\
\text { indicators and alarms. } \\
\text { Over pressurization controls } \\
\text { from reactor (P2). } \\
\text { H/H interlock to ISCO pump } \\
\text { Switch to tempered water }\end{array}$ & 1.00E-01 & $\mathrm{P} 2$ & PRV*PRV & $2.40 \mathrm{E}-10$ & 3 & IV & $\begin{array}{l}\text { Conservative } \\
\text { ignores process } \\
\text { indicators- } \\
\text { assumed fast } \\
\text { acting. Would } \\
\text { require additional } \\
\text { failure (P2) to } \\
\text { challenge pressure } \\
\text { boundary. }\end{array}$ \\
\hline
\end{tabular}


Supplemental Hazard Analysis and Risk Assessment - Hydrotreater

\begin{tabular}{|c|c|c|c|c|c|c|c|c|c|}
\hline Hazardous Event & $\begin{array}{c}\text { Representative } \\
\text { Events }\end{array}$ & $\begin{array}{c}\text { Hazard Controls } \\
\text { Bold }=\text { new selected control } \\
\text { Italics }=\begin{array}{c}\text { existing control newly } \\
\text { assigned }\end{array}\end{array}$ & $\begin{array}{l}\text { Initiating } \\
\text { Event } \\
\text { Frequency }\end{array}$ & $\begin{array}{l}\text { (Enabling) } \\
\text { Process } \\
\text { Control } \\
\text { Failures }\end{array}$ & $\begin{array}{l}\text { Critical } \\
\text { Controls }\end{array}$ & $\begin{array}{c}\text { Event } \\
\text { Frequency }\end{array}$ & $\begin{array}{l}\text { Consequence } \\
\text { Severity }\end{array}$ & $\begin{array}{l}\text { Risk } \\
\text { Bin }\end{array}$ & Comments \\
\hline $\begin{array}{l}\text { Catastrophic } \\
\text { failure of vessel } \\
\text { due to a slow } \\
\text { block in flow path } \\
\text { (PVB) }\end{array}$ & $\begin{array}{l}\text { H. } 4-12 \\
\text { H. } 4-13 \\
\text { H. } 4-16 \\
\text { H. } 4-21 \\
\text { U-26 }\end{array}$ & $\begin{array}{l}\text { L/L mas flow alarm and an } \\
\text { interlock to shut off hydrogen } \\
\text { and ISCO pump (OP2) } \\
\text { Procedural monitoring of system } \\
\text { Sufficient time for operator } \\
\text { response } \\
\text { Process indicators } \\
\text { Design of high pressure } \\
\text { components } \\
\text { Pressure Relief Valve PSC-4002 } \\
\text { (PRV) } \\
\text { Level control on V-140. } \\
\text { Pressure indicators and alarms } \\
\text { (OP2) }\end{array}$ & 1.00E-01 & OP2 & PRV & $1.00 \mathrm{E}-06$ & 3 & IV & \\
\hline
\end{tabular}




\begin{tabular}{|c|c|c|c|c|c|c|c|c|c|}
\hline Hazardous Event & $\begin{array}{l}\text { Representative } \\
\text { Events }\end{array}$ & $\begin{array}{c}\text { Hazard Controls } \\
\text { Bold }=\text { new selected control } \\
\text { Italics }=\text { existing control newly } \\
\text { assigned }\end{array}$ & $\begin{array}{l}\text { Initiating } \\
\text { Event } \\
\text { Frequency }\end{array}$ & $\begin{array}{l}\text { (Enabling) } \\
\text { Process } \\
\text { Control } \\
\text { Failures }\end{array}$ & $\begin{array}{l}\text { Critical } \\
\text { Controls }\end{array}$ & $\begin{array}{c}\text { Event } \\
\text { Frequency }\end{array}$ & $\begin{array}{l}\text { Consequence } \\
\text { Severity }\end{array}$ & $\begin{array}{l}\text { Risk } \\
\text { Bin }\end{array}$ & Comments \\
\hline \multirow[t]{2}{*}{$\begin{array}{l}\text { Failure of vessel } \\
\text { material or piping } \\
\text { system leading } \\
\text { to flammable } \\
\text { atmosphere }\end{array}$} & \multirow[t]{2}{*}{$\begin{array}{l}\text { H. } 4-8 \\
\text { H. } 4-15 \\
\text { H. } 4-19 \\
\text { H. } 4-20 \\
\text { H. } 7-8\end{array}$} & \multirow{2}{*}{$\begin{array}{l}\text { R-130 Design } \\
\text { Procedural step: vessel } \\
\text { inspection, preventative } \\
\text { maintenance and pressure test } \\
\text { Steam impingement } \\
\text { shield/pathway (Graylock and } \\
\text { expanded metal screen/ acrylic } \\
\text { shield on walkway north side of } \\
\text { flange). } \\
\text { Overpressure over-temperature } \\
\text { alarms and controls } \\
\text { Enclosure design - Class } 1 \\
\text { Division } 2 \\
\text { Enclosure ventilation system } \\
\text { (VENT) } \\
\text { Hydrogen monitors on skid and } \\
\text { in enclosure . } \\
\text { Flammable vapor monitor on } \\
\text { skid } \\
\text { Thermowell is replaceable } \\
\text { Robust design for glass } \\
\text { Design of high pressure } \\
\text { components } \\
\text { Design V-125 } 155 \text { psi MAWP, V- } \\
125 \text { PRV set at } \leq 100 \text { psi. } \\
\text { Minimize time in enclosure } \\
\text { (OP1) }\end{array}$} & 1.00E-02 & IGN2(EN) & VENT & 5.00E-06 & 3 & IV & $\begin{array}{l}\text { IE due to leak in } \\
\text { vessel set at LK2 } \\
\text { bounds LK3 } \\
\text { Ignores gas } \\
\text { detection (sudden } \\
\text { event). }\end{array}$ \\
\hline & & & $5.00 \mathrm{E}-03$ & OP1 & & $5.00 \mathrm{E}-04$ & 2 & IV & $\begin{array}{l}\text { Flashing steam } \\
\text { event; IE frequency } \\
\text { reflects required } \\
\text { orientation of spray } \\
\text { and proximity of } \\
\text { operator. } \\
\text { Recommendation: } \\
\text { evaluate use of } \\
\text { PPE for protection } \\
\text { against steam } \\
\text { release events. }\end{array}$ \\
\hline
\end{tabular}




\begin{tabular}{|c|c|c|c|c|c|c|c|c|c|}
\hline Hazardous Event & $\begin{array}{l}\text { Representative } \\
\text { Events }\end{array}$ & $\begin{array}{c}\text { Hazard Controls } \\
\text { Bold = new selected control } \\
\text { Italics = existing control newly } \\
\text { assigned }\end{array}$ & $\begin{array}{l}\text { Initiating } \\
\text { Event } \\
\text { Frequency }\end{array}$ & $\begin{array}{l}\text { (Enabling) } \\
\text { Process } \\
\text { Control } \\
\text { Failures }\end{array}$ & $\begin{array}{l}\text { Critical } \\
\text { Controls }\end{array}$ & $\begin{array}{c}\text { Event } \\
\text { Frequency }\end{array}$ & $\begin{array}{l}\text { Consequence } \\
\text { Severity }\end{array}$ & $\begin{array}{l}\text { Risk } \\
\text { Bin }\end{array}$ & Comments \\
\hline $\begin{array}{l}\text { Gasket failure } \\
\text { leading to } \\
\text { flammable } \\
\text { atmosphere }\end{array}$ & $\begin{array}{l}\text { H.4-26 } \\
\text { H.4-28 }\end{array}$ & $\begin{array}{l}\text { Design of vent system. } \\
\text { Procedural step calibration of } \\
\text { WTM. } \\
\text { Enclosure design - Class } 1 \\
\text { Division } 2 \\
\text { Enclosure ventilation system } \\
\text { (VENT). } \\
\text { Hydrogen monitors on skid and } \\
\text { in enclosure. } \\
\text { Flammable vapor monitor on } \\
\text { skid (GD). } \\
\text { Fire suppression system in } \\
\text { enclosure. } \\
\text { Containment pan on skid and by } \\
\text { the enclosure. } \\
\text { Pressure test procedure. } \\
\text { Design of V-160 A/B and piping. } \\
\text { Drains are double valved, valved } \\
\text { and capped, or valve and quick } \\
\text { disconnect. } \\
\text { Personal Oxygen/ Gas } \\
\text { Detectors would notify } \\
\text { operators of release }\end{array}$ & 1.00E-01 & IGN2(EN) & GD*VENT & 2.65E-06 & 3 & IV & $\begin{array}{l}\text { IE - LK1 leak from } \\
\text { seal } \\
\text { Included GD }\end{array}$ \\
\hline $\begin{array}{l}\text { Operator error - } \\
\text { failure to close a } \\
\text { valve before } \\
\text { opening another } \\
\text { resulting in } \\
\text { flammable } \\
\text { atmosphere }\end{array}$ & H.6-1 & $\begin{array}{l}\text { HV-2018 Action: Replace T } \\
\text { upstream HV-2009 with 3-way } \\
\text { valve. } \\
\text { Check Valve (CK-2007) } \\
\text { Procedural step. }\end{array}$ & 1.00E-01 & & $\begin{array}{l}\text { Prevented } \\
\text { with } \\
\text { 3-way } \\
\text { valve }\end{array}$ & Prevented & & NA & $\begin{array}{l}\text { I.E.F. due to } \\
\text { operator error } \\
\text { Event prevented } \\
\text { due to design } \\
\text { change }\end{array}$ \\
\hline
\end{tabular}




\begin{tabular}{|c|c|c|c|c|c|c|c|c|c|}
\hline Hazardous Event & $\begin{array}{l}\text { Representative } \\
\text { Events }\end{array}$ & $\begin{array}{c}\text { Hazard Controls } \\
\text { Bold = new selected control } \\
\text { Italics = existing control newly } \\
\text { assigned }\end{array}$ & $\begin{array}{l}\text { Initiating } \\
\text { Event } \\
\text { Frequency }\end{array}$ & $\begin{array}{l}\text { (Enabling) } \\
\text { Process } \\
\text { Control } \\
\text { Failures }\end{array}$ & $\begin{array}{l}\text { Critical } \\
\text { Controls }\end{array}$ & $\begin{array}{c}\text { Event } \\
\text { Frequency }\end{array}$ & $\begin{array}{l}\text { Consequence } \\
\text { Severity }\end{array}$ & $\begin{array}{l}\text { Risk } \\
\text { Bin }\end{array}$ & Comments \\
\hline $\begin{array}{l}\text { Operator error - } \\
\text { failure in } \\
\text { procedure } \\
\text { resulting in } \\
\text { flammable } \\
\text { atmosphere }\end{array}$ & $\begin{array}{l}\text { H.1-8 } \\
\text { H.1-12 } \\
\text { H.1-14 } \\
\text { H.2-9 }\end{array}$ & $\begin{array}{l}\text { Pressure test procedures } \\
\text { (detection of no pressure on } \\
\text { system components). } \\
\text { Hydrogen monitors on skid and } \\
\text { in enclosure (GD) } \\
\text { Enclosure Design - Class } 1 \text { Div } \\
2 \text { (IGN1(EN)). } \\
\text { Enclosure Ventilation System } \\
\text { (VENT). } \\
\text { Flammable vapor monitor on } \\
\text { skid } \\
\text { Low pressure alarm - R-130. }\end{array}$ & $1.00 \mathrm{E}-01$ & $\begin{array}{l}\text { IGN1(EN)* } \\
\text { LK1 }\end{array}$ & VENT & $1.00 \mathrm{E}-07$ & 3 & IV & $\begin{array}{l}\text { High pressure } \\
\text { spray of (jet flame) } \\
\text { combustible liquid } \\
\text { and potential } \\
\text { flammable } \\
\text { atmosphere } \\
\text { creation may be } \\
\text { mitigated by steam. }\end{array}$ \\
\hline $\begin{array}{l}\text { Operator error - } \\
\text { failure to close } \\
\text { valve (HV-2009) } \\
\text { resulting in } \\
\text { creation of } \\
\text { spill/spray of } \\
\text { flammable liquid }\end{array}$ & $\begin{array}{l}\text { H.2-17 } \\
\text { H. } 7-9\end{array}$ & $\begin{array}{l}\text { Procedural check that drain } \\
\text { valve is closed. } \\
\text { Enclosure Design - Class } 1 \\
\text { Division } 2 . \\
\text { Enclosure ventilation. } \\
\text { Containment for skid. } \\
\text { Personal Oxygen/ Gas } \\
\text { Detectors would notify } \\
\text { operators of release. }\end{array}$ & 1.00E-01 & IGN1(EN) & & 1.00E-04 & 2 & IV & $\begin{array}{l}\text { Used lower value } \\
\text { for IGN1(EN) due } \\
\text { to small size of spill } \\
\text { \& ventilation } \\
\text { H.2-17 assumes 3- } \\
\text { way valve turned to } \\
\text { bypass condition. }\end{array}$ \\
\hline $\begin{array}{l}\text { Operator error- } \\
\text { failure to purge } \\
\text { resulting in } \\
\text { flammable } \\
\text { atmosphere }\end{array}$ & $\begin{array}{l}\mathrm{H} .8-2 \\
\mathrm{H} .8-3\end{array}$ & $\begin{array}{l}\text { Pressure indicator on reactor. } \\
\text { Procedural step: verify zero } \\
\text { energy- and hold point. } \\
\text { Enclosure ventilation system } \\
\text { (VENT). } \\
\text { Flammable vapor monitor on } \\
\text { skid (GD). } \\
\text { Hydrogen monitor on skid and in } \\
\text { enclosure (GD). }\end{array}$ & 1.00E-01 & IGN2(EN) & VENT*GD & 2.65E-06 & 2 & IV & $\begin{array}{l}\text { Defines transition } \\
\text { to non-Class 1/Div } \\
2 \text { (H.8-3). } \\
\text { Nitrogen flush } \\
\text { performed prior to } \\
\text { disassembly } \\
\text { (possibly between } \\
\text { step } 6 \& 7 \text { ). }\end{array}$ \\
\hline
\end{tabular}




\begin{tabular}{|c|c|c|c|c|c|c|c|c|c|}
\hline Hazardous Event & $\begin{array}{c}\text { Representative } \\
\text { Events }\end{array}$ & $\begin{array}{c}\text { Hazard Controls } \\
\text { Bold }=\text { new selected control } \\
\text { Italics }=\text { existing control newly } \\
\text { assigned }\end{array}$ & $\begin{array}{l}\text { Initiating } \\
\text { Event } \\
\text { Frequency }\end{array}$ & $\begin{array}{l}\text { (Enabling) } \\
\text { Process } \\
\text { Control } \\
\text { Failures }\end{array}$ & $\begin{array}{l}\text { Critical } \\
\text { Controls }\end{array}$ & $\begin{array}{c}\text { Event } \\
\text { Frequency }\end{array}$ & $\begin{array}{l}\text { Consequence } \\
\text { Severity }\end{array}$ & $\begin{array}{c}\text { Risk } \\
\text { Bin }\end{array}$ & Comments \\
\hline $\begin{array}{l}\text { Operator error - } \\
\text { failure to connect } \\
\text { lines resulting in } \\
\text { flammable } \\
\text { atmosphere }\end{array}$ & $\begin{array}{l}\text { H.5-2 } \\
\text { H.7-5 }\end{array}$ & $\begin{array}{l}\text { Design of quick disconnects } \\
\text { (closed unless properly } \\
\text { engaged)(QD). } \\
\text { Enclosure design - Class } 1 \text { Div } 2 \\
\text { (IGN1(EN)). } \\
\text { Enclosure ventilation. } \\
\text { Containment for skids and } \\
\text { enclosure. } \\
\text { Flammable vapor monitoring on } \\
\text { skid. } \\
\text { Fire suppression system in } \\
\text { enclosure. } \\
\text { Procedural step: Connect vent } \\
\text { line prior to nitrogen outlet. }\end{array}$ & 1.00E-01 & $\begin{array}{l}\text { IGN1(EN)* } \\
\text { QD }\end{array}$ & & 2.10E-05 & 2 & IV & $\begin{array}{l}\text { QD failure bounds } \\
\text { line failures. }\end{array}$ \\
\hline $\begin{array}{l}\text { Operator error - } \\
\text { failure to } \\
\text { establish and } \\
\text { maintain Class } 1 \\
\text { Div } 2 \\
\text { environment }\end{array}$ & $\begin{array}{l}\text { H.2-1 } \\
\text { H.2-2 } \\
\text { H.2-3 }\end{array}$ & $\begin{array}{l}\text { Procedural steps: pressure test, } \\
\text { use of anti-sparking tools, initiate } \\
\text { purge. } \\
\text { Enclosure ventilation system } \\
\text { (VENT). } \\
\text { Hydrogen monitors on skid and } \\
\text { in enclosure. } \\
\text { Flammable vapor monitor on } \\
\text { skid. } \\
\text { Pressure monitoring/alarms on } \\
\text { cabinets and furnace enclosure. }\end{array}$ & 1.00E-01 & $\begin{array}{l}\text { LK1* } \\
\text { IGN2(EN) }\end{array}$ & VENT & 5.00E-06 & 2 & IV & \\
\hline $\begin{array}{l}\text { Operator error - } \\
\text { closure of HV- } \\
2006 \text { resulting in } \\
\text { spray of } \\
\text { flammable liquid }\end{array}$ & $\begin{array}{l}\text { H. 2-15 } \\
\text { H.7-7 }\end{array}$ & $\begin{array}{l}\text { Transfer line (tubing) rated at } \\
4800 \text { psi (LK2). } \\
\text { ISCO pump high pressure set } \\
\text { point (process controlled)(P2). } \\
\text { ISCO pump firmware maximum } \\
\text { pressure of } 3750 \text { psi (P2). } \\
\text { ISCO pump shear pin at } 4500 \\
\text { psi (P2). }\end{array}$ & 1.00E-01 & $\begin{array}{l}\text { IGN2(EN)*P2 } \\
\text { *LK2 }\end{array}$ & & 3.00E-05 & 2 & IV & $\begin{array}{l}\text { Requires additional } \\
\text { failure }(\mathrm{P} 2) \text { to } \\
\text { challenge pressure } \\
\text { boundary }\end{array}$ \\
\hline
\end{tabular}




\begin{tabular}{|c|c|c|c|c|c|c|c|c|c|}
\hline Hazardous Event & $\begin{array}{l}\text { Representative } \\
\text { Events }\end{array}$ & $\begin{array}{c}\text { Hazard Controls } \\
\text { Bold = new selected control } \\
\text { Italics = existing control newly } \\
\text { assigned }\end{array}$ & $\begin{array}{l}\text { Initiating } \\
\text { Event } \\
\text { Frequency }\end{array}$ & $\begin{array}{l}\text { (Enabling) } \\
\text { Process } \\
\text { Control } \\
\text { Failures }\end{array}$ & $\begin{array}{l}\text { Critical } \\
\text { Controls }\end{array}$ & $\begin{array}{c}\text { Event } \\
\text { Frequency }\end{array}$ & $\begin{array}{l}\text { Consequence } \\
\text { Severity }\end{array}$ & $\begin{array}{l}\text { Risk } \\
\text { Bin }\end{array}$ & Comments \\
\hline $\begin{array}{l}\text { Failure of } \\
\text { pressure } \\
\text { boundary } \\
\text { (PCV-4007) } \\
\text { resulting in } \\
\text { flammable } \\
\text { atmosphere }\end{array}$ & $\begin{array}{l}\text { H.4-23 } \\
\text { H.4-24 }\end{array}$ & $\begin{array}{l}\text { The badger valve (PV-4005) } \\
\text { would try to control the pressure } \\
\text { (PCV). } \\
\text { PSV-5001 set at } 10 \text { psi (PRV). } \\
\text { Vent system flow path design } \\
\text { (1/2 or greater SS tubing). } \\
\text { Low alarm at PIT-4005 and PIT- } \\
3001 . \\
\text { Design of vent system. } \\
\text { Enclosure Design - Class } 1 \text { Div } \\
2 . \\
\text { Enclosure ventilation system. }\end{array}$ & $1.00 \mathrm{E}-01$ & $\begin{array}{l}\text { IGN2(EN)* } \\
\text { PCV }\end{array}$ & PRV & 3.14E-07 & 3 & IV & $\begin{array}{l}\text { IE due to operator } \\
\text { error (open } \\
\text { bypass) }\end{array}$ \\
\hline $\begin{array}{l}\text { Operational } \\
\text { upset from other } \\
\text { PDL-West } \\
\text { operations } \\
\text { leading to } \\
\text { flammable } \\
\text { atmosphere in } \\
\text { PDL }\end{array}$ & U-31 & $\begin{array}{l}\text { Operational restriction on crane } \\
\text { use } \\
\text { Excess flow valve on gas supply } \\
\text { (EFV) }\end{array}$ & 1.00E-03 & IGN2(EN) & EFV & $6.50 \mathrm{E}-06$ & 3 & IV & $\begin{array}{l}\text { IE based on Admin } \\
\text { control on crane to } \\
\text { restrict usage } \\
\text { during hydrotreater } \\
\text { operation }\end{array}$ \\
\hline $\begin{array}{l}\text { Operational } \\
\text { upset from other } \\
\text { PDL-West } \\
\text { operations } \\
\text { leading to } \\
\text { flammable } \\
\text { atmosphere in } \\
\text { enclosure }\end{array}$ & U-32 & $\begin{array}{l}\text { Flammable vapor monitor } \\
\text { Enclosure Design - Class } 1 \\
\text { Division } 2 \\
\text { MCA alarm, } 20 \text { minute bypass } \\
\text { timer until Scenario A is initiated } \\
\text { (unless operator bypasses) }\end{array}$ & 1.00E-01 & IGN1(EN) & GD & 5.30E-06 & 3 & IV & $\begin{array}{l}\text { Lower frequency } \\
\text { used for IGN1(EN) } \\
\text { due to } \\
\text { concentration of } \\
\text { flammable gas } \\
\text { being drawn into } \\
\text { enclosure. }\end{array}$ \\
\hline
\end{tabular}




\begin{tabular}{|c|c|c|c|c|c|c|c|c|c|}
\hline Hazardous Event & $\begin{array}{l}\text { Representative } \\
\text { Events }\end{array}$ & $\begin{array}{c}\text { Hazard Controls } \\
\text { Bold = new selected control } \\
\text { Italics }=\text { existing control newly } \\
\text { assigned }\end{array}$ & $\begin{array}{l}\text { Initiating } \\
\text { Event } \\
\text { Frequency }\end{array}$ & $\begin{array}{l}\text { (Enabling) } \\
\text { Process } \\
\text { Control } \\
\text { Failures }\end{array}$ & $\begin{array}{l}\text { Critical } \\
\text { Controls }\end{array}$ & $\begin{array}{c}\text { Event } \\
\text { Frequency }\end{array}$ & $\begin{array}{l}\text { Consequence } \\
\text { Severity }\end{array}$ & $\begin{array}{c}\text { Risk } \\
\text { Bin }\end{array}$ & Comments \\
\hline \multirow[t]{2}{*}{$\begin{array}{l}\text { Hydrogen leak } \\
\text { resulting in } \\
\text { flammable } \\
\text { atmosphere }\end{array}$} & \multirow[t]{2}{*}{$U-24$} & \multirow{2}{*}{$\begin{array}{l}\text { Design of hydrogen pipe system } \\
\text { (one piece of tubing ( } 17 \mathrm{ft}) \\
\text { rated at maximum pressure; } \\
\text { Located in a tray sitting in a tube } \\
\text { holder supported across the } \\
\text { length. It is a } 13 \text { foot elevation, } \\
\text { in a tray, protected from edge of } \\
\text { building. } \\
\text { Excess flow valve on hydrogen } \\
\text { supply (catastrophic break) } \\
\text { (EFV) } \\
\text { Makeup air unit for enclosure. } \\
\text { Passive PDL roof vents (PDL) }\end{array}$} & 2.93E-06 & IGN2(EN) & EFV & 1.90E-08 & 3 & IV & $\begin{array}{l}\text { IE set at PC1 } \\
\text { assuming } \\
\text { catastrophic pipe } \\
\text { failure }\end{array}$ \\
\hline & & & 2.93E-05 & IGN2(EN) & PDL & $1.47 \mathrm{E}-06$ & 3 & IV & $\begin{array}{l}\text { IE reflects a PC1 } \\
\text { leak that is large } \\
\text { enough to pose } \\
\text { concern but small } \\
\text { enough to not be } \\
\text { detectable by } \\
\text { operators or } \\
\text { activate EFV. } \\
\text { Smaller leaks pose } \\
\text { a concern similar to } \\
\text { other users of } \\
\text { hydrogen } \\
\text { (addressed within } \\
\text { HDI/SMPs) and } \\
\text { would most likely } \\
\text { not reach } \\
\text { significant } \\
\text { concentrations due } \\
\text { to passive PDL } \\
\text { roof vents }\end{array}$ \\
\hline $\begin{array}{l}\text { Failure in } \\
\text { ventilation } \\
\text { resulting in } \\
\text { flammable } \\
\text { atmosphere }\end{array}$ & $\begin{array}{l}U-3 \\
U-4 \\
U-5 \\
U-6\end{array}$ & $\begin{array}{l}\text { Exhaust flow switch initiates } \\
\text { Scenario A on loss of flow } \\
\text { Design of stack } \\
\text { Loss of ventilation fan trips } \\
\text { process controls }\end{array}$ & $1.00 \mathrm{E}-01$ & $\begin{array}{l}\text { IGN1(EN)* } \\
\text { LK1 }\end{array}$ & P3 & 1.00E-06 & 2 & IV & \\
\hline $\begin{array}{l}\text { Activation of } \\
\text { pressure relief } \\
\text { resulting in } \\
\text { flammable } \\
\text { atmosphere }\end{array}$ & U-8 & Design of knockout pot and lines & 1.47E-02 & IGN3(EN) & & 1.47E-05 & 1 & IV & $\begin{array}{l}\text { IE based on relief } \\
\text { flow path open to } \\
\text { atmosphere, } \\
\text { personnel impacts } \\
\text { not expected. }\end{array}$ \\
\hline
\end{tabular}




\begin{tabular}{|c|c|c|c|c|c|c|c|c|c|}
\hline Hazardous Event & $\begin{array}{c}\text { Representative } \\
\text { Events }\end{array}$ & $\begin{array}{c}\text { Hazard Controls } \\
\text { Bold }=\text { new selected control } \\
\text { Italics }=\text { existing control newly } \\
\text { assigned }\end{array}$ & $\begin{array}{l}\text { Initiating } \\
\text { Event } \\
\text { Frequency }\end{array}$ & $\begin{array}{l}\text { (Enabling) } \\
\text { Process } \\
\text { Control } \\
\text { Failures }\end{array}$ & $\begin{array}{l}\text { Critical } \\
\text { Controls }\end{array}$ & $\begin{array}{c}\text { Event } \\
\text { Frequency }\end{array}$ & $\begin{array}{c}\text { Consequence } \\
\text { Severity }\end{array}$ & $\begin{array}{c}\text { Risk } \\
\text { Bin }\end{array}$ & Comments \\
\hline $\begin{array}{l}\text { Operator Error } \\
\text { during pressure } \\
\text { check results in } \\
\text { release of } \\
\text { nitrogen into } \\
\text { enclosure. } \\
\text { (Frequency } \\
\text { bounds N2 line } \\
\text { failures). } \\
\text { Possible release } \\
\text { of H2S }\end{array}$ & $\begin{array}{l}\text { H.1-7 } \\
\text { H.1-10 } \\
\text { H.1-20 } \\
\text { H.2-24 } \\
\text { H.2-25 } \\
\text { H.4-33 } \\
\text { H.5-3 } \\
\text { H.8-1 } \\
\text { U-29 }\end{array}$ & $\begin{array}{l}\text { Procedural step: pressure test, } \\
\text { valve lineup, pressure indication. } \\
\text { Enclosure Ventilation System } \\
\text { operable when system is } \\
\text { pressurized (VENT). } \\
\text { Procedural STEP - 1) any leak } \\
\text { testing of the system (with } \\
\text { nitrogen) will be conducted } \\
\text { with the ventilation system } \\
\text { operational and 2) the nitrogen } \\
\text { supply will be closed when not } \\
\text { in use (OP2). } \\
\text { Design of pressure vessels, } \\
\text { components, and enclosure. } \\
\text { Low pressure alarms. } \\
\text { PCV-2001 sets pressure to 10 } \\
\text { psi. } \\
\text { Overpressure relief supply } \\
\text { system PCV-2030. } \\
\text { Overpressure vented through LP } \\
\text { header. } \\
\text { Flammable vapor/hydrogen } \\
\text { monitor on skid. } \\
\text { Personal Oxygen/ Gas } \\
\text { Detectors would notify } \\
\text { operators of release. }\end{array}$ & 1.00E-01 & OP2 & VENT & 5.00E-06 & 3 & IV & $\begin{array}{l}\text { See Distillation } \\
\text { Column } \\
\text { Supplemental } \\
\text { Report Appendix A, } \\
\text { Item } 4 \text { for O2 } \\
\text { calculation } \\
\text { performed w \& w/o } \\
\text { ventilation. }\end{array}$ \\
\hline Fire in PDL-West & U-15 & $\begin{array}{l}\text { Fire alarm system activation } \\
\text { initiates Scenario A. } \\
\text { Operator Response to Fire } \\
\text { Alarm. }\end{array}$ & 1.00E-01 & OP2 & P3 & 5.00E-05 & 2 & IV & $\begin{array}{l}\text { Frequency is the } \\
\text { point at which } \\
\text { system would be } \\
\text { challenged. }\end{array}$ \\
\hline
\end{tabular}




\begin{tabular}{|c|c|c|c|c|c|c|c|c|c|}
\hline Hazardous Event & $\begin{array}{c}\text { Representative } \\
\text { Events }\end{array}$ & $\begin{array}{c}\text { Hazard Controls } \\
\text { Bold }=\text { new selected control } \\
\text { Italics }=\text { existing control newly } \\
\text { assigned }\end{array}$ & $\begin{array}{l}\text { Initiating } \\
\text { Event } \\
\text { Frequency }\end{array}$ & $\begin{array}{l}\text { (Enabling) } \\
\text { Process } \\
\text { Control } \\
\text { Failures }\end{array}$ & $\begin{array}{l}\text { Critical } \\
\text { Controls }\end{array}$ & $\begin{array}{c}\text { Event } \\
\text { Frequency }\end{array}$ & $\begin{array}{l}\text { Consequence } \\
\text { Severity }\end{array}$ & $\begin{array}{c}\text { Risk } \\
\text { Bin }\end{array}$ & Comments \\
\hline \multirow{2}{*}{$\begin{array}{l}\text { High Pressure } \\
\text { Leak in the ISCO } \\
\text { pump resulting in } \\
\text { spray or spill of } \\
\text { bio-oil and } \\
\text { hydrogen. }\end{array}$} & $\begin{array}{l}\mathrm{H} .4-4 \\
\mathrm{H} .4-5\end{array}$ & $\begin{array}{l}\text { Gasket } \\
\text { Pressure Boundary } \\
\text { Secondary containment for small } \\
\text { leaks. } \\
\text { Auto-shutdown of pumps for }\end{array}$ & 1.00E-01 & $\mathrm{P} 1$ & VENT & 3.00E-05 & 2 & IV & \multirow{2}{*}{$\begin{array}{l}\text { Minimize time in } \\
\text { enclosure. } \\
\text { For small leaks the } \\
\text { consequence } \\
\text { would be reduced } \\
\text { to } 1 .\end{array}$} \\
\hline & & $\begin{array}{l}\text { large leaks (P1). } \\
\text { Enclosure ventilation (VENT). } \\
\text { Personal Oxygen/ Gas } \\
\text { Detectors would notify } \\
\text { operators of release. }\end{array}$ & 1.00E-01 & & VENT & 1.00E-04 & 1 & IV & \\
\hline $\begin{array}{l}\text { Medium } \\
\text { Pressure leaks } \\
\text { of Product } \\
\text { Failure to open } \\
\text { vent line on } \\
\text { Product Tank } \\
\text { resulting in } \\
\text { bio-oil/ dirty } \\
\text { water spray. }\end{array}$ & H.3-5 & $\begin{array}{l}\text { LG-147 visual indication during } \\
\text { operator rounds. } \\
\text { Product scales on V-160 and } \\
\text { V-161. } \\
\text { Local indication on wet } \\
\text { WTM-152 test meter. } \\
\text { Relief valves on product gas } \\
\text { header at } 10 \text { psi. } \\
\text { Relief Valves on V-160A/B and } \\
\text { V-161A/B set at } 70 \text { psi. } \\
\text { Relief Valve on LG-147 feed line } \\
\text { at } 70 \text { psi. }\end{array}$ & $1.00 \mathrm{E}-01$ & LK2 & & $1.00 \mathrm{E}-03$ & 2 & IV & $\begin{array}{l}\text { Pressure should } \\
\text { equalize (PRV on } \\
\text { LG-147 } 70 \text { psi, } \\
\text { PRV } \\
\text { V-160/161 } 70 \text { psi) }\end{array}$ \\
\hline
\end{tabular}




\begin{tabular}{|c|c|c|c|c|c|c|c|c|c|}
\hline Hazardous Event & $\begin{array}{l}\text { Representative } \\
\text { Events }\end{array}$ & $\begin{array}{c}\text { Hazard Controls } \\
\text { Bold = new selected control } \\
\text { Italics = existing control newly } \\
\text { assigned }\end{array}$ & $\begin{array}{l}\text { Initiating } \\
\text { Event } \\
\text { Frequency }\end{array}$ & $\begin{array}{l}\text { (Enabling) } \\
\text { Process } \\
\text { Control } \\
\text { Failures }\end{array}$ & $\begin{array}{l}\text { Critical } \\
\text { Controls }\end{array}$ & $\begin{array}{c}\text { Event } \\
\text { Frequency }\end{array}$ & $\begin{array}{l}\text { Consequence } \\
\text { Severity }\end{array}$ & $\begin{array}{c}\text { Risk } \\
\text { Bin }\end{array}$ & Comments \\
\hline $\begin{array}{l}\text { Low Pressure } \\
\text { leaks of bio- } \\
\text { oil/sulfiding } \\
\text { agent }\end{array}$ & $\begin{array}{l}\text { H.1-13 } \\
\text { H.1-22 } \\
\text { H.1-23 } \\
\text { H.1-24 } \\
\text { H.1-25 } \\
\text { H.1-28 } \\
\text { H.2-8 } \\
\text { H.2-16 } \\
\text { H.2-22 } \\
\text { H.2-23 }\end{array}$ & $\begin{array}{l}\text { Procedural step (OP2) } \\
\text { Secondary Containment } \\
\text { Air system pressure set at } 100 \\
\text { psi. } \\
\text { Filters rated at } 250 \mathrm{psi} \\
\text { V-120 rated at } 135 \mathrm{psi} \\
\text { PRV set at } 70 \text { psi } \\
\text { Transfer line rated at >3000 psi } \\
\text { V-120 level indicator } \\
\text { High-High interlocks } \\
\text { Bypass system } \\
\text { Vessel Design }\end{array}$ & 1.00E-02 & OP2 & & 5.00E-04 & 2 & IV & $\begin{array}{l}\text { Secondary } \\
\text { containment and } \\
\text { enclosure } \\
\text { ventilation. PPE } \\
\text { will further reduce } \\
\text { consequence. } \\
\text { IE reflects } \\
\text { significant } \\
\text { leak/spray from } \\
\text { pressurized } \\
\text { system. For } \\
\text { failure associated } \\
\text { with open lines } \\
\text { consequence } \\
\text { would be } 1 .\end{array}$ \\
\hline $\begin{array}{l}\text { Low Pressure } \\
\text { leaks of Aqueous } \\
\text { (water } \\
\text { by-product) }\end{array}$ & $\begin{array}{l}\text { H.4-31a } \\
\text { H.4-32 }\end{array}$ & $\begin{array}{l}\text { Pressure test procedure (OP2) } \\
\text { Vessel and piping design } \\
\text { Quick disconnect } \\
\text { Containment }\end{array}$ & 1.00E-02 & OP2 & & 5.00E-04 & 1 & IV & $\begin{array}{l}\text { Secondary } \\
\text { containment and } \\
\text { enclosure } \\
\text { ventilation. PPE } \\
\text { will further reduce } \\
\text { consequence. }\end{array}$ \\
\hline
\end{tabular}




\begin{tabular}{|c|c|c|c|c|c|c|c|c|c|}
\hline Hazardous Event & $\begin{array}{l}\text { Representative } \\
\text { Events }\end{array}$ & $\begin{array}{c}\text { Hazard Controls } \\
\text { Bold = new selected control } \\
\text { Italics }=\text { existing control newly } \\
\text { assigned }\end{array}$ & $\begin{array}{l}\text { Initiating } \\
\text { Event } \\
\text { Frequency }\end{array}$ & $\begin{array}{l}\text { (Enabling) } \\
\text { Process } \\
\text { Control } \\
\text { Failures }\end{array}$ & $\begin{array}{l}\text { Critical } \\
\text { Controls }\end{array}$ & $\begin{array}{c}\text { Event } \\
\text { Frequency }\end{array}$ & $\begin{array}{c}\text { Consequence } \\
\text { Severity }\end{array}$ & $\begin{array}{c}\text { Risk } \\
\text { Bin }\end{array}$ & Comments \\
\hline $\begin{array}{l}\text { Catastrophic } \\
\text { failure of vessel } \\
\text { given inability of } \\
\text { pressure relief } \\
\text { due to blocked } \\
\text { low pressure } \\
\text { flow path }\end{array}$ & $U-7$ & $\begin{array}{l}\text { Administrative controls to } \\
\text { check relief header - Modify } \\
\text { the SOP to require verification } \\
\text { that the low pressure relief line is } \\
\text { free of obstruction } \\
\text { Design of system includes bird } \\
\text { screens, double release path at } \\
\text { top. Design pressure of } \\
\text { knockout pot is } 12 \text { psi. }\end{array}$ & 1.00E-02 & $\begin{array}{l}\text { PROCESS* } \\
\text { OP2 }\end{array}$ & & $5.00 \mathrm{E}-06$ & 3 & IV & $\begin{array}{l}\text { IE due to blocked } \\
\text { flow path (e.g., } \\
\text { mud daubers). } \\
\text { Event frequency } \\
\text { requires multiple } \\
\text { failures to correct } \\
\text { the blockage and } \\
\text { upset/failures } \\
\text { (0.01) resulting in } \\
\text { activation of PRV. } \\
\text { Failure point is } \\
\text { most likely in low } \\
\text { pressure portion of } \\
\text { system- in LP } \\
\text { header (knockout } \\
\text { pot or stack) }\end{array}$ \\
\hline
\end{tabular}




\section{Appendix A: Valve Calculation}

The critical characteristics of high/low pressure interface valves (flow coefficient; CV) were obtained and maximum flow rates were calculated as an action item to ensure appropriate controls and design elements were in place to mitigate the following identified hazardous events.

1. H.1-11 and H.1-21 involve an operator error or component failure; opening of valve that exposes low pressure piping to a high system pressure. The limiting failure for LG-147 (liquid) is LV-4003 failing open, while limiting failure for WTM-152 (vent) is PV-4005 failing open. The maximum normal system pressure is 2500 psig.

- LV-4003 has a max CV of 0.001 (0.0625" orifice); at 2500 psig, this will allow a liquid flow of $\sim 0.05 \mathrm{gpm}$. Immediately downstream is a 70 psig relief valve (PSV-4016, 0.138" orifice); Zeton has sized this valve such that it is sufficient to relieve this flowrate.

- PV-4005 has a max CV of 0.03 (0.0860" orifice); at 2500 psig, this will allow a hydrogen flow of $\sim 135 \mathrm{scfm}$. Immediately downstream is a 10 psig relief valve (PSV-5001); at 18 psig, it will relieve $\sim 135$ SCFM of hydrogen flow. This provides adequate protection for WTM-152, which has a 1.5 atm ( 22 psig) pressure rating.

2. H.4-23 and H.4-24 involve the failure of a pressure control valve (PV-4005).

- PV-4005 has a max CV of 0.03 (0.0860" orifice); at 2500 psig, this will allow a hydrogen flow of $\sim 135 \mathrm{scfm}$. Immediately downstream is a 10 psig relief valve (PSV-5001); Zeton has sized this valve such that it is sufficient to relieve this flowrate at 18 psig, it will relieve $\sim 135$ SCFM of hydrogen flow; this provides adequate protection for WTM-152, which has a $1.5 \mathrm{~atm}(\sim 22 \mathrm{psig})$ pressure rating\}. 


\section{Appendix B. Chemical Safety Data Sheets}

\begin{tabular}{|c|c|}
\hline Chemical Name & Safety Data Sheet (SDS) \\
\hline Di-tert-butyl disulfide & SDS-DTBS \\
\hline Decane & SDS-Decane \\
\hline Acetone & SDS-Acetone \\
\hline
\end{tabular}

